\title{
Reduction of the parameters in MSSM
}

\author{
S. Heinemeyer, ${ }^{a, b, c}$ M. Mondragón, ${ }^{d}$ N. Tracas ${ }^{e, f}$ and G. Zoupanos ${ }^{e, f, g}$ \\ ${ }^{a}$ Instituto de Física Teórica (UAM/CSIC), Universidad Autonóma de Madrid, \\ Ciudad Universitaria de Cantoblanco, E-28049 Madrid, Spain \\ ${ }^{b}$ Campus of International Excellence $U A M+C S I C$, \\ Cantoblanco, E-28049 Madrid, Spain \\ ${ }^{c}$ Instituto de Física de Cantabria (CSIC-UC), University of Cantabria, \\ Av. de los Castros, E-39005 Santander, Spain \\ ${ }^{d}$ Instituto de Física, Universidad Nacional Autónoma de México, \\ A.P. 20-364, CDMX 01000, México \\ e Theoretical Physics Department, CERN, \\ Geneva, Switzerland \\ ${ }^{f}$ Physics Department, Nat. Technical University, \\ 15780 Zografou, Athens, Greece \\ ${ }^{g}$ Max-Planck Institut für Physik, \\ Föhringer Ring 6, D-80805 München, Germany \\ E-mail: Sven.Heinemeyer@cern.ch, myriam@fisica.unam.mx, \\ ntrac@central.ntua.gr, George.Zoupanos@cern.ch
}

ABSTRACT: In the present work we search for renormalization group invariant relations among the various massless and massive parameters of the Minimal Supersymmetric Standard Model. We find that indeed several of the previously free parameters of the model can be reduced in favor of few, the unique gauge coupling and the gaugino mass at the unification scale among them. Taking into account the various experimental constraints, including the $B$-physics ones, we predict the Higgs and the supersymmetric spectrum. We find that the lightest Higgs mass is in comfortable agreement with the measured value and its experimental and theoretical uncertainties, while the electroweak supersymmetric spectrum starts at $1.3 \mathrm{TeV}$ and the colored at $\sim 4 \mathrm{TeV}$. Thus the reduced MSSM is in natural agreement with all LHC measurements and searches. The supersymmetric and heavy Higgs particles will likely escape the detection at the LHC, as well as at ILC and CLIC. However, the FCC-hh will be able to fully test the predicted parameter space.

KeYwords: Renormalization Group, Supersymmetric Gauge Theory

ArXiv EPrint: 1712.02729 


\section{Contents}

1 Introduction 1

2 Reduction of parameters $\quad 4$

$\begin{array}{lll}3 & \text { Reduction of dimensionless parameters in the MSSM } & 7\end{array}$

4 Reduction of dimensionful parameters in the MSSM 9

5 Phenomenological constraints $\quad 12$

$\begin{array}{lll}5.1 \text { Flavor constraints } & 12\end{array}$

$\begin{array}{ll}5.2 & \text { The light Higgs boson mass } \\ \end{array}$

$\begin{array}{llr}6 & \text { Numerical analysis } & 14\end{array}$

$\begin{array}{lll}7 & \text { Conclusions } & 19\end{array}$

\section{Introduction}

The main expectation of the particle physics community from a unified description of the observed interactions is to understand the present day large number of free parameters of the Standard Model (SM) in terms of a few fundamental ones. In other words, to achieve reduction of parameters at a fundamental level.

The traditional way to reduce the number of free parameters of a theory, which in turn would make it more predictive, is to introduce a symmetry. Grand Unified Theories (GUTs) are very good examples of this strategy [1-5]. In the case of minimal SU(5), because of the (approximate) gauge coupling unification, it was possible to reduce the gauge couplings of the SM and give a prediction for one of them. In fact, the LEP data [6] were interpreted as suggesting that a further symmetry, namely $N=1$ global supersymmetry (SUSY) $[7,8]$ should also be required to make the prediction viable. GUTs can also relate the Yukawa couplings among themselves, again $\mathrm{SU}(5)$ provided an example of this by predicting the ratio $M_{\tau} / M_{b}[9]$ in the SM. Unfortunately, requiring more symmetry does not necessarily helps, since additional complications are introduced due to new degrees of freedom that normally are needed, requiring in turn new ways and channels of breaking the symmetry, among others, which in general reduce the predictivity of a theory.

A natural extension of the GUT idea is to find a way to relate the gauge and Yukawa sectors of a theory, that is to achieve Gauge-Yukawa Unification (GYU). A symmetry which naturally relates the two sectors is SUSY, in particular $N=2$ SUSY [10]. However, $N=2$ supersymmetric theories have serious phenomenological problems due to light mirror fermions. Other theories such as superstring theories or composite models might provide 
relations among the gauge and Yukawa couplings, but have even more phenomenological problems. A successful strategy in relating dimensionless couplings has been developed in a series of studies [11-24]. It was based on searches for renormalization group invariant (RGI) relations. This program, called Gauge-Yukawa unification scheme, applied in the dimensionless couplings of supersymmetric GUTs, such as gauge and Yukawa couplings, had already celebrated successes by predicting correctly, among others, the top quark mass in the finite and in the minimal $N=1$ supersymmetric SU(5) GUTs [14-16], SU(3) ${ }^{3}[20$ 23] and later in the Minimal Supersymmetric Standard Model (MSSM) [24]. One of the impressive aspects of the RGI relations is that their validity can be guaranteed to all-orders in perturbation theory by studying the uniqueness of the resulting relations at one-loop, as was proven $[25,26]$ in the early days of the program of reduction of couplings [25-30]. Even more impressive is the fact that it is possible to find RGI relations among couplings guaranteeing finiteness to all-orders in perturbation theory [31-35].

SUSY seems to be an essential ingredient for a plenomenologically successful realization of the above strategy. Nevertheless its breaking has to be understood too in order to extend the successes in other sectors of the theory, such as the Higgs masses and the SUSY spectrum.

Indeed, the search for RGI relations has been extended to the soft SUSY-breaking sector (SSB) of these theories [19, 36], which involves parameters of dimension one and two. The first important development in this programme concerned the combined reduction of couplings and masses in supersymmetric theories [19]. In this work the coefficients of the soft SUSY-breaking terms were reduced in order to minimize the number of independent parameters. The scheme of dimensional renormalization was used with mass parameters introduced similarly to couplings. Then the differential equations of the renormalization group also involve derivatives with respect to the masses. It is characteristic for dimensional renormalization that those $\beta$-functions which carry a dimension are linear or quadratic forms in the dimensional couplings and masses, while the coefficients of these polynomials depend on the dimensionless couplings only. Since in this approach the mass parameters enter similarly to the couplings, masses are included with the couplings in the reduction process. In this way non-trivial constraints on the soft SUSY-breaking terms were obtained which are compatible with renormalization and lead to surprisingly simple sum rules [37].

Another very important development concerning the renormalization properties of the SSB was made in refs. [38-44], based conceptually and technically on the work of ref. [45]: the powerful supergraph method [46-49] for studying supersymmetric theories was applied to the softly broken ones by using the "spurion" external space-time independent superfields [50]. In the latter method a softly broken supersymmetric gauge theory is considered as a supersymmetric one in which the various parameters such as couplings and masses have been promoted to external superfields that acquire "vacuum expectation values". Based on this method the relations among the soft term renormalization and that of an unbroken supersymmetric theory were derived. In particular the $\beta$-functions of the parameters of the softly broken theory are expressed in terms of partial differential operators involving the dimensionless parameters of the unbroken theory. The key point in the strategy of refs. [41-44] in solving the set of coupled differential equations so as to be able to 
express all parameters in a RGI way, was to transform the partial differential operators involved to total derivative operators. This is indeed possible to be done on the RGI surface which is defined by the solution of the reduction equations. The last has very important consequences in the finite theories since the finiteness of the dimensionless sector can be transferred to the SSB sector too.

In parallel to the above theoretical developments certain phenomenological issues have been established too. For long time a rather constrained universal set of soft scalar masses has been assumed in the SSB sector of supersymmetric theories not only for economy and simplicity but for a number of other reason too: (a) they were part of the constraints that preserve finiteness up to two-loops [51, 52], (b) they are RGI up to two-loops in more general supersymmetric gauge theories, subject to the condition known as $P=1 / 3 Q[36]$ (where all relevant details and definitions can be found), and (c) they appear in the attractive dilaton dominated SUSY-breaking superstring scenarios [53-55]. However, further studies have shown that there exist a number of technical problems all due to the fact that the universality assumption for the soft scalar masses is very restrictive. For instance, (i) in finite unified theories the universality predicts that the lightest supersymmetric particle is a charged particle, namely the superpartner of the $\tau$-lepton, (ii) the standard radiative electroweak symmetry breaking of the MSSM does not work with universal soft scalar masses [55], and (iii) which is more serious, the universal soft scalar masses lead to charge and/or color breaking minima deeper than the standard vacuum [56]. In addition criticisms arose on an aesthetic basis, i.e. that the universal assumption is too strong to be put by hand given that it does not result from something fundamental. A way out was indirectly already suggested in ref [19], where the solutions found among soft scalar masses were very different from the universal one. Moreover a more careful look suggested the existence of a "sum rule" among the soft scalar masses and the gaugino mass. This interesting observation was clearly done in ref [37] where it was examined in $N=1$ Gauge-Yukawa unified theories at one-loop for the non-finite case and then at two-loops for the finite case [57]. The sum rule manages to overcome all the unpleasant phenomenological consequences mentioned above. Moreover it was proven [44] that the sum rule for the soft scalar masses is RGI to all-orders for both the general as well as for the finite case. Finally, the exact $\beta$-function for the soft scalar masses in the Novikov-Shifman-Vainstein-Zakharov (NSVZ) scheme [58-60] for the softly broken supersymmetric QCD has been obtained [44].

Using the above tools and results it was possible to study and predict the spectrum of the full finite models in terms of few input parameters. A particular finite model was selected out of this examination and provided us with the prediction for the lightest MSSM Higgs boson in the range of $121-126 \mathrm{GeV}[61,63-83]^{1}$ four and half years before the experimental discovery $[84,85] .{ }^{2}$ Identifying the lightest Higgs boson with the newly discovered state one can restrict the allowed parameter space of the model. A similar analysis was done for the reduced MSSM [24].

\footnotetext{
${ }^{1}$ See also a short version of [61] in ref. [62].

${ }^{2}$ It should be kept in mind that this prediction did not yet include the resummation of large logarithmic contributions to the light Higgs boson mass, see the discussion in section 5 .
} 
In the present work we examine the reduced MSSM using the "exact" relations among soft scalar and gaugino masses, following the original analysis suggested in ref [19]. Obviously the reduced MSSM in the present case is much more constrained as compared to the previous one [24], which was enjoying the benefit of the relaxed "sum rule". The results are confronted with the relevant flavor physics results. We evaluate the full SUSY spectrum (for sfermions restricted to the third generation), which turns out to be rather heavy, and in particular we calculate the lightest MSSM Higgs-boson mass. Here, in contrast to previous evaluations, an improved calculation is employed that yields more reliable results for heavy SUSY masses. The light Higgs-boson mass is naturally found in the region of $124-129 \mathrm{GeV}$.

\section{Reduction of parameters}

The reduction of couplings was originally formulated for massless theories on the basis of the Callan-Symanzik equation $[25,26]$. The extension to theories with massive parameters is not straightforward if one wants to keep the generality and the rigor on the same level as for the massless case; one has to fulfill a set of requirements coming from the renormalization group equations, the Callan-Symanzik equations, etc. along with the normalization conditions imposed on irreducible Green's functions [86]. There has been a lot of progress in this direction starting from ref. [19], as it is already mentioned in the Introduction, where it was assumed that a mass-independent renormalization scheme could be employed so that all the RG functions have only trivial dependencies on dimensional parameters and then the mass parameters were introduced similarly to couplings (i.e. as a power series in the couplings). This choice was justified later in $[87,88]$ where the scheme independence of the reduction principle has been proven generally, i.e it was shown that apart from dimensionless couplings, pole masses and gauge parameters, the model may also involve coupling parameters carrying a dimension and masses. Therefore here, to simplify the analysis, we follow ref. [19] and we too use a mass-independent renormalization scheme.

We start by considering a renormalizable theory which contain a set of $(N+1)$ dimension-zero couplings, $\left(\hat{g}_{0}, \hat{g}_{1}, \ldots, \hat{g}_{N}\right)$, a set of $L$ parameters with mass-dimension one, $\left(\hat{h}_{1}, \ldots, \hat{h}_{L}\right)$, and a set of $M$ parameters with mass-dimension two, $\left(\hat{m}_{1}^{2}, \ldots, \hat{m}_{M}^{2}\right)$. The renormalized irreducible vertex function $\Gamma$ satisfies the RG equation

$$
\mathcal{D} \Gamma\left[\Phi^{\prime} s ; \hat{g}_{0}, \hat{g}_{1}, \ldots, \hat{g}_{N} ; \hat{h}_{1}, \ldots, \hat{h}_{L} ; \hat{m}_{1}^{2}, \ldots, \hat{m}_{M}^{2} ; \mu\right]=0
$$

where

$$
\mathcal{D}=\mu \frac{\partial}{\partial \mu}+\sum_{i=0}^{N} \beta_{i} \frac{\partial}{\partial \hat{g}_{i}}+\sum_{a=1}^{L} \gamma_{a}^{h} \frac{\partial}{\partial \hat{h}_{a}}+\sum_{\alpha=1}^{M} \gamma_{\alpha}^{m^{2}} \frac{\partial}{\partial \hat{m}_{\alpha}^{2}}+\sum_{J} \Phi_{I} \gamma_{J}^{\phi I} \frac{\delta}{\delta \Phi_{J}}
$$

where $\mu$ is the energy scale, while $\beta_{i}$ are the $\beta$-functions of the various dimensionless couplings $g_{i}, \Phi_{I}$ are the various matter fields and $\gamma_{\alpha}^{m^{2}}, \gamma_{a}^{h}$ and $\gamma_{J}^{\phi I}$ are the mass, trilinear coupling and wave function anomalous dimensions, respectively (where $I$ enumerates the 
matter fields). In a mass independent renormalization scheme, the $\gamma$ 's are given by

$$
\begin{aligned}
\gamma_{a}^{h} & =\sum_{b=1}^{L} \gamma_{a}^{h, b}\left(g_{0}, g_{1}, \ldots, g_{N}\right) \hat{h}_{b}, \\
\gamma_{\alpha}^{m^{2}} & =\sum_{\beta=1}^{M} \gamma_{\alpha}^{m^{2}, \beta}\left(g_{0}, g_{1}, \ldots, g_{N}\right) \hat{m}_{\beta}^{2}+\sum_{a, b=1}^{L} \gamma_{\alpha}^{m^{2}, a b}\left(g_{0}, g_{1}, \ldots, g_{N}\right) \hat{h}_{a} \hat{h}_{b},
\end{aligned}
$$

where $\gamma_{a}^{h, b}, \gamma_{\alpha}^{m^{2}, \beta}$ and $\gamma_{\alpha}^{m^{2}, a b}$ are power series of the $g$ 's (which are dimensionless) in perturbation theory.

We look for a reduced theory where

$$
g \equiv g_{0}, \quad h_{a} \equiv \hat{h}_{a} \quad \text { for } 1 \leq a \leq P, \quad m_{\alpha}^{2} \equiv \hat{m}_{\alpha}^{2} \quad \text { for } 1 \leq \alpha \leq Q
$$

are independent parameters and the reduction of the parameters left

$$
\begin{aligned}
\hat{g}_{i} & =\hat{g}_{i}(g), & (i=1, \ldots, N), \\
\hat{h}_{a} & =\sum_{b=1}^{P} f_{a}^{b}(g) h_{b}, & (a=P+1, \ldots, L), \\
\hat{m}_{\alpha}^{2} & =\sum_{\beta=1}^{Q} e_{\alpha}^{\beta}(g) m_{\beta}^{2}+\sum_{a, b=1}^{P} k_{\alpha}^{a b}(g) h_{a} h_{b}, & (\alpha=Q+1, \ldots, M)
\end{aligned}
$$

is consistent with the RG equations (2.1), (2.2). It turns out that the following relations should be satisfied

$$
\begin{gathered}
\beta_{g} \frac{\partial \hat{g}_{i}}{\partial g}=\beta_{i}, \quad(i=1, \ldots, N), \\
\beta_{g} \frac{\partial \hat{h}_{a}}{\partial g}+\sum_{b=1}^{P} \gamma_{b}^{h} \frac{\partial \hat{h}_{a}}{\partial h_{b}}=\gamma_{a}^{h}, \quad(a=P+1, \ldots, L), \\
\beta_{g} \frac{\partial \hat{m}_{\alpha}^{2}}{\partial g}+\sum_{a=1}^{P} \gamma_{a}^{h} \frac{\partial \hat{m}_{\alpha}^{2}}{\partial h_{a}}+\sum_{\beta=1}^{Q} \gamma_{\beta}^{m^{2}} \frac{\partial \hat{m}_{\alpha}^{2}}{\partial m_{\beta}^{2}}=\gamma_{\alpha}^{m^{2}}, \quad(\alpha=Q+1, \ldots, M) .
\end{gathered}
$$

Using eqs. (2.3) and (2.4), the above relations reduce to

$$
\begin{gathered}
\beta_{g} \frac{d f_{a}^{b}}{d g}+\sum_{c=1}^{P} f_{a}^{c}\left[\gamma_{c}^{h, b}+\sum_{d=P+1}^{L} \gamma_{c}^{h, d} f_{d}^{b}\right]-\gamma_{a}^{h, b}-\sum_{d=P+1}^{L} \gamma_{a}^{h, d} f_{d}^{b}=0, \\
(a=P+1, \ldots, L ; b=1, \ldots, P), \\
\beta_{g} \frac{d e_{\alpha}^{\beta}}{d g}+\sum_{\gamma=1}^{Q} e_{\alpha}^{\gamma}\left[\gamma_{\gamma}^{m^{2}, \beta}+\sum_{\delta=Q+1}^{M} \gamma_{\gamma}^{m^{2}, \delta} e_{\delta}^{\beta}\right]-\gamma_{\alpha}^{m^{2}, \beta}-\sum_{\delta=Q+1}^{M} \gamma_{\alpha}^{m^{2}, d} e_{\delta}^{\beta}=0, \\
(\alpha=Q+1, \ldots, M q \beta=1, \ldots, Q),
\end{gathered}
$$




$$
\begin{array}{r}
\beta_{g} \frac{d k_{\alpha}^{a b}}{d g}+2 \sum_{c=1}^{P}\left(\gamma_{c}^{h, a}+\sum_{d=P+1}^{L} \gamma_{c}^{h, d} f_{d}^{a}\right) k_{\alpha}^{c b}+\sum_{\beta=1}^{Q} e_{\alpha}^{\beta}\left[\gamma_{\beta}^{m^{2}, a b}+\sum_{c, d=P+1}^{L} \gamma_{\beta}^{m^{2}, c d} f_{c}^{a} f_{d}^{b}\right. \\
\left.+2 \sum_{c=P+1}^{L} \gamma_{\beta}^{m^{2}, c b} f_{c}^{a}+\sum_{\delta=Q+1}^{M} \gamma_{\beta}^{m^{2}, d} k_{\delta}^{a b}\right]-\left[\gamma_{\alpha}^{m^{2}, a b}+\sum_{c, d=P+1}^{L} \gamma_{\alpha}^{m^{2}, c d} f_{c}^{a} f_{d}^{b}\right. \\
\left.+2 \sum_{c=P+1}^{L} \gamma_{\alpha}^{m^{2}, c b} f_{c}^{a}+\sum_{\delta=Q+1}^{M} \gamma_{\alpha}^{m^{2}, \delta} k_{\delta}^{a b}\right]=0 \\
(\alpha=Q+1, \ldots, M ; a, b=1, \ldots, P)
\end{array}
$$

The above relations ensure that the irreducible vertex function of the reduced theory

$$
\begin{array}{r}
\Gamma_{R}\left[\Phi ' s ; g ; h_{1}, \ldots, h_{P} ; m_{1}^{2}, \ldots, m_{Q}^{2} ; \mu\right] \equiv \\
\equiv \Gamma\left[\Phi ' s ; g, \hat{g}_{1}(g) \ldots, \hat{g}_{N}(g) ; h_{1}, \ldots, h_{P}, \hat{h}_{P+1}(g, h), \ldots, \hat{h}_{L}(g, h) ;\right. \\
\left.m_{1}^{2}, \ldots, m_{Q}^{2}, \hat{m}_{Q+1}^{2}\left(g, h, m^{2}\right), \ldots, \hat{m}_{M}^{2}\left(g, h, m^{2}\right) ; \mu\right]
\end{array}
$$

has the same renormalization group flow as the original one.

The assumptions that the reduced theory is perturbatively renormalizable means that the functions $\hat{g}_{i}, f_{a}^{b}, e_{\alpha}^{\beta}$ and $k_{\alpha}^{a b}$, defined in (2.4), should be expressed as a power series in the primary coupling $g$ :

$$
\begin{aligned}
& \hat{g}_{i}=g \sum_{n=0}^{\infty} \rho_{i}^{(n)} g^{n}, \quad f_{a}^{b}=g \sum_{n=0}^{\infty} \eta_{a}^{b(n)} g^{n} \\
& e_{\alpha}^{\beta}=\sum_{n=0}^{\infty} \xi_{\alpha}^{\beta(n)} g^{n}, \quad k_{\alpha}^{a b}=\sum_{n=0}^{\infty} \chi_{\alpha}^{a b(n)} g^{n} .
\end{aligned}
$$

The above expansion coefficients can be found by inserting these power series into eqs. (2.5), (2.6) and requiring the equations to be satisfied at each order of $g$. It should be noted that the existence of a unique power series solution is a non-trivial matter: it depends on the theory as well as on the choice of the set of independent parameters.

It should also be noted that in the case that there are no independent mass-dimension 1 parameters $(\hat{h})$ the reduction of these terms take naturally the form

$$
\hat{h}_{a}=\sum_{b=1}^{L} f_{a}^{b}(g) M,
$$

where $M$ is a mass-dimension 1 parameter which could be a gaugino mass which corresponds to the independent (gauge) coupling. In case, on top of that, there are no independent mass-dimension 2 parameters $\left(\hat{m}^{2}\right)$, the corresponding reduction takes analogous form

$$
\hat{m}_{a}^{2}=\sum_{b=1}^{M} e_{a}^{b}(g) M^{2} .
$$




\section{Reduction of dimensionless parameters in the MSSM}

Hereafter we are working in the framework of MSSM, assuming though the existence of a covering GUT. The superpotential of the MSSM (where again we restrict ourselves to the third generation of sfermions) is defined by

$$
W=Y_{t} H_{2} Q t^{c}+Y_{b} H_{1} Q b^{c}+Y_{\tau} H_{1} L \tau^{c}+\mu H_{1} H_{2},
$$

where $Q, L, t, b, \tau, H_{1}, H_{2}$ are the usual superfields of MSSM, while the SSB Lagrangian is given by

$$
\begin{aligned}
-\mathcal{L}_{\mathrm{SSB}}= & \sum_{\phi} m_{\phi}^{2} \phi^{*} \phi+\left[m_{3}^{2} H_{1} H_{2}+\sum_{i=1}^{3} \frac{1}{2} M_{i} \lambda_{i} \lambda_{i}+\text { h.c }\right] \\
& +\left[h_{t} H_{2} Q t^{c}+h_{b} H_{1} Q b^{c}+h_{\tau} H_{1} L \tau^{c}+\text { h.c. }\right]
\end{aligned}
$$

where $\phi$ represents the scalar component of all superfields, $\lambda$ refers to the gaugino fields while in the last brace we refer to the scalar components of the corresponding superfield. The Yukawa $Y_{t, b, \tau}$ and the trilinear $h_{t, b, \tau}$ couplings refer to the third generator only, neglecting the first two generations.

Let us start with the dimensionless couplings, i.e. gauge and Yukawa. As a first step we consider only the strong coupling and the top and bottom Yukawa couplings, while the other two gauge couplings and the tau Yukawa will be treated as corrections. Following the above line, we reduce the Yukawa couplings in favor of the strong coupling $\alpha_{3}$

$$
\frac{Y_{i}^{2}}{4 \pi} \equiv \alpha_{i}=G_{i}^{2} \alpha_{3}, \quad i=t, b,
$$

and using the RGE for the Yukawa, we get

$$
G_{i}^{2}=\frac{1}{3}, \quad i=t, b .
$$

This system of the top and bottom Yukawa couplings reduced with the strong one is dictated by (i) the different running behaviour of the $\mathrm{SU}(2)$ and $\mathrm{U}(1)$ coupling compared to the strong one [89] and (ii) the incompatibility of applying the above reduction for the tau Yukawa since the corresponding $G^{2}$ turns negative [24]. Adding now the two other gauge couplings and the tau Yukawa in the RGE as corrections, we obtain

$$
G_{t}^{2}=\frac{1}{3}+\frac{71}{525} \rho_{1}+\frac{3}{7} \rho_{2}+\frac{1}{35} \rho_{\tau}, \quad G_{b}^{2}=\frac{1}{3}+\frac{29}{525} \rho_{1}+\frac{3}{7} \rho_{2}-\frac{6}{35} \rho_{\tau}
$$

where

$$
\rho_{1,2}=\frac{g_{1,2}^{2}}{g_{3}^{2}}=\frac{\alpha_{1,2}}{\alpha_{3}}, \quad \rho_{\tau}=\frac{g_{\tau}^{2}}{g_{3}^{2}}=\frac{\frac{Y_{\tau}^{2}}{4 \pi}}{\alpha_{3}}
$$

Note that the corrections in eq. (3.3) are taken at the GUT scale and under the assumption that

$$
\frac{d}{d g_{3}}\left(\frac{Y_{t, b}^{2}}{g_{3}^{2}}\right)=0
$$


Let us comment further on our assumption above, which led to the eq. (3.3). In practice we assume that even including the corrections from the rest of the gauge as well as the tau Yukawa couplings, at the GUT scale the ratio of the top and bottom couplings $\alpha_{t, b}$ over the strong coupling are still constant, i.e. their scale dependence is negligible. Or, rephrasing it, our assumption can be understood as a requirement that in the ultraviolet (close to the GUT scale) the ratios of the top and bottom Yukawa couplings over the strong coupling become least sensitive against the change of the renormalization scale. This requirement sets the boundary condition at the GUT scale, given in eq. (3.3). Alternatively one could follow the systematic method to include the corrections to a non-trivially reduced system developed in ref [91], but considering two reduced systems: the first one consisting of the "top, bottom" couplings and the second of the "strong, bottom" ones. We plan to return with the full analysis of the latter possibility, including the dimensionful parameters, in a future publication.

In the next order the corrections are assumed to be in the form

$$
\alpha_{i}=G_{i}^{2} \alpha_{3}+J_{i}^{2} \alpha_{3}^{2}, \quad i=t, b .
$$

Then, the coefficients $J_{i}$ are given by

$$
J_{i}^{2}=\frac{1}{4 \pi} \frac{17}{24}, \quad i=t, b
$$

for the case where only the strong gauge and the top and bottom Yukawa couplings are active, while for the case where the other two gauge and the tau Yukawa couplings are added as corrections we obtain

$$
J_{t}^{2}=\frac{1}{4 \pi} \frac{N_{t}}{D}, \quad J_{b}^{2}=\frac{1}{4 \pi} \frac{N_{b}}{5 D},
$$

where

$$
\begin{array}{rl}
D=2 & 7250\left(196000+44500 \rho_{1}+2059 \rho_{1}^{2}+200250 \rho_{2}+22500 \rho_{1} \rho_{2}+50625 \rho_{2}^{2}\right. \\
& \left.-33375 \rho_{\tau}-5955 \rho_{1} \rho_{\tau}-16875 \rho_{2} \rho_{\tau}-1350 \rho_{\tau}^{2}\right), \\
N_{t}=- & -35714875000-10349167500 \rho_{1}+21077903700 \rho_{1}^{2}+9057172327 \rho_{1}^{3} \\
& +481651575 \rho_{1}^{4}-55566000000 \rho_{2}+2857680000 \rho_{1} \rho_{2}+34588894725 \rho_{1}^{2} \rho_{2} \\
& +5202716130 \rho_{1}^{3} \rho_{2}+3913875000 \rho_{2}^{2}+8104595625 \rho_{1} \rho_{2}^{2}+11497621500 \rho_{1}^{2} \rho_{2}^{2} \\
& +27047671875 \rho_{2}^{3}+1977918750 \rho_{1} \rho_{2}^{3}+7802578125 \rho_{2}^{4}+3678675000 \rho_{\tau} \\
& +1269418500 \rho_{1} \rho_{\tau}-2827765710 \rho_{1}^{2} \rho_{\tau}-1420498671 \rho_{1}^{3} \rho_{\tau}+7557637500 \rho_{2} \rho_{\tau} \\
& -2378187000 \rho_{1} \rho_{2} \rho_{\tau}-4066909425 \rho_{1}^{2} \rho_{2} \rho_{\tau}-1284018750 \rho_{2}^{2} \rho_{\tau}-1035973125 \rho_{1} \rho_{2}^{2} \rho_{\tau} \\
& -2464171875 \rho_{2}^{3} \rho_{\tau}+1230757500 \rho_{\tau}^{2}+442136100 \rho_{1} \rho_{\tau}^{2}-186425070 \rho_{1}^{2} \rho_{\tau}^{2} \\
& +1727460000 \rho_{2} \rho_{\tau}^{2}+794232000 \rho_{1} \rho_{2} \rho_{\tau}^{2}+973518750 \rho_{2}^{2} \rho_{\tau}^{2} \\
& \left.-325804500 \rho_{\tau}^{3}-126334800 \rho_{1} \rho_{\tau}^{3}-412695000 \rho_{2} \rho_{\tau}^{3}-32724000 \rho_{\tau}^{4}\right), \\
N_{b}=- & -178574375000-71734162500 \rho_{1}+36055498500 \rho_{1}^{2}+13029194465 \rho_{1}^{3} \\
& +977219931 \rho_{1}^{4}-277830000000 \rho_{2}-69523650000 \rho_{1} \rho_{2}+72621383625 \rho_{1}^{2} \rho_{2}
\end{array}
$$




$$
\begin{aligned}
& +10648126350 \rho_{1}^{3} \rho_{2}+19569375000 \rho_{2}^{2}+13062459375 \rho_{1} \rho_{2}^{2}+25279672500 \rho_{1}^{2} \rho_{2}^{2} \\
& +135238359375 \rho_{2}^{3}+16587281250 \rho_{1} \rho_{2}^{3}+39012890625 \rho_{2}^{4}+58460062500 \rho_{\tau} \\
& +35924411250 \rho_{1} \rho_{\tau}-13544261325 \rho_{1}^{2} \rho_{\tau}-2152509435 \rho_{1}^{3} \rho_{\tau}-13050843750 \rho_{2} \rho_{\tau} \\
& +45805646250 \rho_{1} \rho_{2} \rho_{\tau}-75889125 \rho_{1}^{2} \rho_{2} \rho_{\tau}-24218578125 \rho_{2}^{2} \rho_{\tau}+17493046875 \rho_{1} \rho_{2}^{2} \rho_{\tau} \\
& -1158046875 \rho_{2}^{3} \rho_{\tau}-36356775000 \rho_{\tau}^{2}-26724138000 \rho_{1} \rho_{\tau}^{2}-4004587050 \rho_{1}^{2} \rho_{\tau}^{2} \\
& -97864200000 \rho_{2} \rho_{\tau}^{2}-22359847500 \rho_{1} \rho_{2} \rho_{\tau}^{2}-39783656250 \rho_{2}^{2} \rho_{\tau}^{2}+25721797500 \rho_{\tau}^{3} \\
& \left.+3651097500 \rho_{1} \rho_{\tau}^{3}+11282287500 \rho_{2} \rho_{\tau}^{3}+927855000 \rho_{\tau}^{4}\right) .
\end{aligned}
$$

\section{Reduction of dimensionful parameters in the MSSM}

We move now to the dimension-1 parameters of the SSB Lagrangian, namely the trilinear couplings $h_{t, b, \tau}$ of the SSB Lagrangian, eq. (3.2). Again, following the pattern in the Yukawa reduction, in the first stage we reduce $h_{t, b}$, while $h_{\tau}$ will be treated as a correction.

$$
h_{i}=c_{i} Y_{i} M_{3}=c_{i} G_{i} M_{3} g_{3}, \quad i=t, b,
$$

where $M_{3}$ is the gluino mass. Using the RGE for the two $h$ we get

$$
c_{t}=c_{b}=-1,
$$

where we have also used the 1-loop relation between the gaugino mass and the gauge coupling RGE

$$
2 M_{i} \frac{d g_{i}}{d t}=g_{i} \frac{d M_{i}}{d t}, \quad i=1,2,3
$$

Adding the other two gauge couplings as well as the tau Yukawa $h_{\tau}$ as correction we get

$$
c_{t}=-\frac{A_{A} A_{b b}+A_{t b} B_{B}}{A_{b t} A_{t b}-A_{b b} A_{t t}}, \quad c_{b}=-\frac{A_{A} A_{b t}+A_{t t} B_{B}}{A_{b t} A_{t b}-A_{b b} A_{t t}},
$$

where

$$
\begin{array}{llrl}
A_{t t}=G_{b}^{2}-\frac{16}{3}-3 \rho_{2}-\frac{13}{15} \rho_{1}, & A_{A}=\frac{16}{3}+3 \rho_{2}^{2}+\frac{13}{15} \rho_{1}^{2} \\
A_{b b}=G_{t}^{2}+\rho_{\tau}-\frac{16}{3}-3 \rho_{2}-\frac{7}{15} \rho_{1}, & B_{B}=\frac{16}{3}+3 \rho_{2}^{2}+\frac{7}{15} \rho_{1}^{2}+\rho_{h_{\tau}} \rho_{\tau}^{1 / 2} \\
A_{t b}=G_{b}^{2}, & A_{b t}=G_{t}^{2}, & \rho_{h_{\tau}}=\frac{h_{\tau}}{g_{3} M_{3}} .
\end{array}
$$

Finally we consider the soft squared masses $m_{\phi}^{2}$ of the SSB Lagrangian. Their reduction, according to the discussion in section 3 , takes the form

$$
m_{i}^{2}=c_{i} M_{3}^{2}, \quad i=Q, u, d, H_{u}, H_{d}
$$


The 1-loop RGE for the scalar masses reduce to the following algebraic system (where we have added the corrections from the two gauge couplings, the tau Yukawa and $h_{\tau}$ )

$$
\begin{aligned}
-12 c_{Q} & =X_{t}+X_{b}-\frac{32}{3}-6 \rho_{2}^{3}-\frac{2}{15} \rho_{1}^{3}+\frac{1}{5} \rho_{1} S, \\
-12 c_{u} & =2 X_{t}-\frac{32}{3}-\frac{32}{15} \rho_{1}^{3}-\frac{4}{5} \rho_{1} S, \\
-12 c_{d} & =2 X_{b}-\frac{32}{3}-\frac{8}{15} \rho_{1}^{3}+\frac{2}{5} \rho_{1} S, \\
-12 c_{H_{u}} & =3 X_{t}-6 \rho_{2}^{3}-\frac{6}{5} \rho_{1}^{3}+\frac{3}{5} \rho_{1} S, \\
-12 c_{H_{d}} & =3 X_{b}+X_{\tau}-6 \rho_{2}^{3}-\frac{6}{5} \rho_{1}^{3}-\frac{3}{5} \rho_{1} S,
\end{aligned}
$$

where

$$
\begin{aligned}
X_{t} & =2 G_{t}^{2}\left(c_{H_{u}}+c_{Q}+c_{u}\right)+2 c_{t}^{2} G_{t}^{2}, \\
X_{b} & =2 G_{b}^{2}\left(c_{H_{d}}+c_{Q}+c_{d}\right)+2 c_{b}^{2} G_{b}^{2}, \\
X_{\tau} & =2 \rho_{\tau} c_{H_{d}}+2 \rho_{h_{\tau}}^{2}, \\
S & =c_{H_{u}}-c_{H_{d}}+c_{Q}-2 c_{u}+c_{d} .
\end{aligned}
$$

Solving the above system for the coefficients $c_{Q, u, d, H_{u}, H_{d}}$ we get

$$
\begin{aligned}
c_{Q} & =-\frac{c_{Q \mathrm{Num}}}{D_{m}}, & c_{u} & =-\frac{1}{3} \frac{c_{u \mathrm{Num}}}{D_{m}}, c_{d}=-\frac{c_{d \mathrm{Num}}}{D_{m}}, \\
c_{H_{u}} & =-\frac{2}{3} \frac{c_{H u \mathrm{Num}}}{D_{m}}, & c_{H_{d}} & =-\frac{c_{H d \mathrm{Num}}}{D_{m}},
\end{aligned}
$$

where

$$
\begin{aligned}
D_{m}= & 4\left(6480+6480 G_{b}^{2}+6480 G_{t}^{2}+6300 G_{b}^{2} G_{t}^{2}+\rho_{1}\left(1836+1836 G_{b}^{2}+1836 G_{t}^{2}+1785 G_{b}^{2} G_{t}^{2}\right)\right. \\
& \left.+\rho_{\tau}\left[1080+540 G_{b}^{2}+1080 G_{t}^{2}+510 G_{b}^{2} G_{t}^{2}+252 \rho_{1}+99 G_{b}^{2} \rho_{1}+252 G_{t}^{2} \rho_{1}+92 G_{b}^{2} G_{t}^{2} \rho_{1}\right]\right), \\
c_{Q \mathrm{Num}}= & 2160 F_{Q}+G_{b}^{2}\left(-360 F_{d}-360 F_{H_{d}}+1800 F_{Q}\right)+G_{t}^{2}\left(-360 F_{H_{u}}+1800 F_{Q}-360 F_{u}\right) \\
& +G_{b}^{2} G_{t}^{2}\left(-300 F_{d}-300 F_{H_{d}}-300 F_{H_{u}}+1500 F_{Q}-300 F_{u}\right) \\
& +\rho_{1}\left(-36 F_{d}+36 F_{H_{d}}-36 F_{H_{u}}+576 F_{Q}+72 F_{u}\right) \\
& +G_{b}^{2} \rho_{1}\left(-138 F_{d}-66 F_{H_{d}}-36 F_{H_{u}}+474 F_{Q}+72 F_{u}\right) \\
& +G_{t}^{2} \rho_{1}\left(-36 F_{d}+36 F_{H_{d}}-138 F_{H_{u}}+474 F_{Q}-30 F_{u}\right) \\
& +G_{b}^{2} G_{t}^{2} \rho_{1}\left(-120 F_{d}-50 F_{H_{d}}-120 F_{H_{u}}+390 F_{Q}-15 F_{u}\right) \\
& +\rho_{\tau}\left[360 F_{Q}+G_{b}^{2}\left(-60 F_{d}+120 F_{Q}\right)+G_{t}^{2}\left(-60 F_{H_{u}}+300 F_{Q}-60 F_{u}\right)\right. \\
& +G_{b}^{2} G_{t}^{2}\left(-50 F_{d}-20 F_{H_{u}}+100 F_{Q}-20 F_{u}\right)+\rho_{1}\left(-6 F_{d}-6 F_{H_{u}}+78 F_{Q}+12 F_{u}\right) \\
& +G_{b}^{2} \rho_{1}\left(-11 F_{d}+22 F_{Q}\right)+G_{t}^{2} \rho_{1}\left(-6 F_{d}-20 F_{H_{u}}+64 F_{Q}-2 F_{u}\right) \\
& \left.+G_{b}^{2} G_{t}^{2} \rho_{1}\left(-9 F_{d}-4 F_{H_{u}}+18 F_{Q}-3 F_{u}\right)\right] \\
= & 6480 F_{u}+6480 F_{u} G_{b}^{2}+G_{t}^{2}\left(-2160 F_{H_{u}}-2160 F_{Q}+4320 F_{u}\right) \\
& +G_{b}^{2} G_{t}^{2}\left(360 F_{d}+360 F_{H_{d}}-2160 F_{H_{u}}-1800 F_{Q}+4140 F_{u}\right) \\
& +\rho_{1}\left(432 F_{d}-432 F_{H_{d}}+432 F_{H_{u}}+432 F_{Q}+972 F_{u}\right)
\end{aligned}
$$




$$
\begin{aligned}
& +G_{b}^{2} \rho_{1}\left(432 F_{d}-432 F_{H_{d}}+432 F_{H_{u}}+432 F_{Q}+972 F_{u}\right) \\
& +G_{t}^{2} \rho_{1}\left(432 F_{d}-432 F_{H_{d}}-180 F_{H_{u}}-180 F_{Q}+360 F_{u}\right) \\
& +G_{b}^{2} G_{t}^{2} \rho_{1}\left(522 F_{d}-318 F_{H_{d}}-192 F_{H_{u}}-90 F_{Q}+333 F_{u}\right) \\
& +\rho_{\tau}\left[1080 F_{u}+540 G_{b}^{2} F_{u}+G_{t}^{2}\left(-360 F_{H_{u}}-360 F_{Q}+720 F_{u}\right)\right. \\
& +G_{b}^{2} G_{t}^{2}\left(60 F_{d}-180 F_{H_{u}}-120 F_{Q}+330 F_{u}\right)+\rho_{1}\left(72 F_{d}+72 F_{H_{u}}+72 F_{Q}+108 F_{u}\right) \\
& +G_{b}^{2} \rho_{1}\left(36 F_{H_{u}}+27 F_{u}\right)+72 G_{t}^{2} \rho_{1}\left(F_{d}-12 F_{H_{u}}-12 F_{Q}+24 F_{u}\right) \\
& \left.+G_{b}^{2} G_{t}^{2} \rho_{1}\left(9 F_{d}+4 F_{H_{u}}-18 F_{Q}+3 F_{u}\right)\right] \text {, } \\
& c_{d \mathrm{Num}}=2160 F_{d}+G_{b}^{2}\left(1440 F_{d}-720 F_{H_{d}}-720 F_{Q}\right)+2160 F_{d} G_{t}^{2} \\
& +G_{b}^{2} G_{t}^{2}\left(1380 F_{d}-720 F_{H_{d}}+120 F_{H_{u}}-600 F_{Q}+120 F_{u}\right) \\
& +\rho_{1}\left(540 F_{d}+72 F_{H_{d}}-72 F_{H_{u}}-72 F_{Q}+144 F_{u}\right) \\
& +G_{b}^{2} \rho_{1}\left(336 F_{d}-132 F_{H_{d}}-72 F_{H_{u}}-276 F_{Q}+144 F_{u}\right) \\
& +G_{t}^{2} \rho_{1}\left(540 F_{d}+72 F_{H_{d}}-72 F_{H_{u}}-72 F_{Q}+144 F_{u}\right) \\
& +G_{b}^{2} G_{t}^{2} \rho_{1}\left(321 F_{d}-134 F_{H_{d}}-36 F_{H_{u}}-240 F_{Q}+174 F_{u}\right) \\
& +\rho_{\tau}\left[360 F_{d}+G_{b}^{2}\left(60 F_{d}-120 F_{Q}\right)+360 F_{d} G_{t}^{2}+G_{b}^{2} G_{t}^{2}\left(50 F_{d}+20 F_{H_{u}}-100 F_{Q}+20 F_{u}\right)\right. \\
& +\rho_{1}\left(72 F_{d}-12 F_{H_{u}}-12 F_{Q}+24 F_{u}\right)+G_{b}^{2} \rho_{1}\left(11 F_{d}-22 F_{Q}\right) \\
& \left.+G_{t}^{2} \rho_{1}\left(72 F_{d}-12 F_{H_{u}}-12 F_{Q}+24 F_{u}\right)+G_{b}^{2} G_{t}^{2} \rho_{1}\left(9 F_{d}+4 F_{H_{u}}-18 F_{Q}+3 F_{u}\right)\right], \\
& c_{H u \mathrm{Num}}=3240 F_{H_{u}}+3240 F_{H_{u}} G_{b}^{2}+G_{t}^{2}\left(1620 F_{H_{u}}-1620 F_{Q}-1620 F_{u}\right) \\
& +G_{b}^{2} G_{t}^{2}\left(270 F_{d}+270 F_{H_{d}}+1530 F_{H_{u}}-1350 F_{Q}-1620 F_{u}\right) \\
& +\rho_{1}\left(-162 F_{d}+162 F_{H_{d}}+756 F_{H_{u}}-162 F_{Q}+324 F_{u}\right) \\
& +G_{b}^{2} \rho_{1}\left(-162 F_{d}+162 F_{H_{d}}+756 F_{H_{u}}-162 F_{Q}+324 F_{u}\right) \\
& +G_{t}^{2} \rho_{1}\left(-162 F_{d}+162 F_{H_{d}}+297 F_{H_{u}}-621 F_{Q}-135 F_{u}\right) \\
& +G_{b}^{2} G_{t}^{2} \rho_{1}\left(-81 F_{d}+234 F_{H_{d}}+276 F_{H_{u}}-540 F_{Q}-144 F_{u}\right) \\
& +\rho_{\tau}\left[540 F_{H_{u}}+270 F_{H_{u}} G_{b}^{2}+G_{t}^{2}\left(270 F_{H_{u}}-270 F_{Q}-270 F_{u}\right)\right. \\
& +G_{b}^{2} G_{t}^{2}\left(45 F_{d}+120 F_{H_{u}}-90 F_{Q}-135 F_{u}\right)+\rho_{1}\left(-27 F_{d}+99 F_{H_{u}}-27 F_{Q}+54 F_{u}\right) \\
& +G_{b}^{2} \rho_{1}\left(36 F_{H_{u}}+27 F_{u}-27 F_{d}\right)+G_{t}^{2} \rho_{1}\left(36 F_{H_{u}}-90 F_{Q}-9 F_{u}\right) \\
& \left.+G_{b}^{2} G_{t}^{2} \rho_{1}\left(9 F_{d}+4 F_{H_{u}}-18 F_{Q}+3 F_{u}\right)\right] \text {, } \\
& c_{H d \mathrm{Num}}=2160 F_{H_{d}}+G_{b}^{2}\left(-1080 F_{d}+1080 F_{H_{d}}-1080 F_{Q}\right)+2160 F_{H_{d}} G_{t}^{2} \\
& +G_{b}^{2} G_{t}^{2}\left(-1080 F_{d}+1020 F_{H_{d}}+180 F_{H_{u}}-900 F_{Q}+180 F_{u}\right) \\
& +\rho_{1}\left(108 F_{d}+504 F_{H_{d}}+108 F_{H_{u}}+108 F_{Q}-216 F_{u}\right) \\
& +G_{b}^{2} \rho_{1}\left(-198 F_{d}+198 F_{H_{d}}+108 F_{H_{u}}-198 F_{Q}-216 F_{u}\right) \\
& +G_{t}^{2} \rho\left(108 F_{d} 1+504 F_{H_{d}}+108 F_{H_{u}}+108 F_{Q}-216 F_{u}\right) \\
& +G_{b}^{2} G_{t}^{2} \rho_{1}\left(-201 F_{d}+184 F_{H_{d}}+156 F_{H_{u}}-150 F_{Q}-159 F_{u}\right)
\end{aligned}
$$

and

$$
\begin{aligned}
F_{Q} & =2 c_{t}^{2} G_{t}^{2}+2 c_{b}^{2} G_{b}^{2}-\frac{32}{3}-6 \rho_{2}^{3}-\frac{2}{15} \rho_{1}^{3}, \\
F_{u} & =4 c_{t}^{2} G_{t}^{2}-\frac{32}{3}-\frac{32}{15} \rho_{1}^{3},
\end{aligned}
$$




$$
\begin{aligned}
F_{d} & =4 c_{b}^{2} G_{b}^{2}-\frac{32}{3}-\frac{8}{15} \rho_{1}^{3}, \\
F_{H_{u}} & =6 c_{t}^{2} G_{t}^{2}-6 \rho_{2}^{3}-\frac{6}{5} \rho_{1}^{3}, \\
F_{H_{d}} & =6 c_{b}^{2} G_{b}^{2}+2 \rho_{h_{\tau}}^{2}-6 \rho_{2}^{3}-\frac{6}{5} \rho_{1}^{3},
\end{aligned}
$$

while $G_{t, b}^{2}, \rho_{1,2, \tau}$ and $\rho_{h_{\tau}}$ has been defined in eqs. (3.3), (3.4), (4.1) respectively. For our completely reduced system, i.e. $g_{3}, Y_{t}, Y_{b}, h_{t}, h_{b}$, the coefficients of the soft masses become

$$
c_{Q}=c_{u}=c_{d}=\frac{2}{3}, \quad c_{H_{u}}=c_{H_{d}}=-1 / 3
$$

obeying the celebrated sum rules

$$
\frac{m_{Q}^{2}+m_{u}^{2}+m_{H_{u}}^{2}}{M_{3}^{2}}=c_{Q}+c_{u}+c_{H_{u}}=1, \quad \frac{m_{Q}^{2}+m_{d}^{2}+m_{H_{d}}^{2}}{M_{3}^{2}}=c_{Q}+c_{d}+c_{H_{d}}=1 .
$$

The $\mu$ parameter of the superpotential cannot be reduced, at least in a simple way of the form $\mu=c_{\mu} M_{3} g_{3}$ as an ansatz at one loop. The parameter $m_{3}^{2}$ in the SSB sector could in principle be reduced in favor of $\mu$ and $M_{3}$, but in our analysis we keep $m_{3}^{2}$ as independent parameter. However, it should be noted that the requirement of radiative electroweak symmetry breaking (EWSB) relates $\mu$ and $m_{3}^{2}$, and leaves only one of them as an independent parameter, which we choose to be $\mu$.

\section{Phenomenological constraints}

In this section we will briefly describe the phenomenological constraints that we apply on the parameter space of the reduced MSSM, as described above.

\subsection{Flavor constraints}

As additional constraints we consider four types of flavor contraints, where SUSY is know to have a possible impact. We consider the flavour observables $\operatorname{BR}(b \rightarrow s \gamma), \operatorname{BR}\left(B_{s} \rightarrow\right.$ $\left.\mu^{+} \mu^{-}\right), \operatorname{BR}\left(B_{u} \rightarrow \tau \nu\right)$ and $\Delta B_{M_{s}}{ }^{3}$ The uncertainties are the linear combination of the experimental error and twice the theoretical uncertainty in the MSSM (if no specific MSSM estimate is avialabe we use the SM uncertainty).

For the branching ratio $\operatorname{BR}(b \rightarrow s \gamma)$, we take the value given by the Heavy Flavour Averaging Group (HFAG) is $[92-97]^{4}$

$$
\frac{\mathrm{BR}(b \rightarrow s \gamma)^{\exp }}{\mathrm{BR}(b \rightarrow s \gamma)^{\mathrm{SM}}}=1.089 \pm 0.27
$$

For the branching ratio $\mathrm{BR}\left(B_{s} \rightarrow \mu^{+} \mu^{-}\right)$we use a combination of CMS and LHCb data [99$106]$

$$
\operatorname{BR}\left(B_{s} \rightarrow \mu^{+} \mu^{-}\right)=(2.9 \pm 1.4) \times 10^{-9}
$$

\footnotetext{
${ }^{3}$ We do not employ the very latest experimental data, but this has a minor impact on our analysis.

${ }^{4}$ With updates of [97] available in ref. [98].
} 
For the $B_{u}$ decay to $\tau \nu$ we use the limit $[97,98,107-109]$

$$
\frac{\mathrm{BR}\left(B_{u} \rightarrow \tau \nu\right)^{\exp }}{\mathrm{BR}\left(B_{u} \rightarrow \tau \nu\right)^{\mathrm{SM}}}=1.39 \pm 0.69
$$

As our final flavor observalbe we include $\Delta M_{B_{s}}$ as $[110,111]$

$$
\frac{\Delta M_{B_{s}}^{\exp }}{\Delta M_{B_{s}}^{\mathrm{SM}}}=0.97 \pm 0.2 .
$$

Our theory evaluations are obtained with the code SuFla $[107,108] .{ }^{5}$

We do not include a bound from the cold dark matter (CDM) density. It is well known that the lightest neutralino, being the lightest supersymmetric particle (LSP) in our model, is an excellent candidate for CDM $[112,113]$. However, the models could easily be extended to contain (a) small R-parity violating term(s) [114-117]. They would have a small impact on the collider phenomenology discussed here (apart from the fact that the SUSY search strategies could not rely on a 'missing energy' signature), but would remove the CDM bound completely. Other mechanisms, not involving R-parity violation (and keeping the 'missing energy' signature), that could be invoked if the amount of CDM appears to be too large, concern the cosmology of the early universe. For instance, "thermal inflation" [118] or "late time entropy injection" [119] could bring the CDM density into agreement with the WMAP measurements. This kind of modifications of the physics scenario neither concerns the theory basis nor the collider phenomenology, but could have a strong impact on the CDM derived bounds. (Lower values than the ones permitted by the experimental measurements are naturally allowed if another particle than the lightest neutralino constitutes CDM.)

We will briefly comment on the anomalous magnetic moment of the muon, $(g-2)_{\mu}$, at the end of section 6 .

\subsection{The light Higgs boson mass}

Due to the fact that the quartic couplings in the Higgs potential are given by the SM gauge couplings, the lightest Higgs boson mass is not a free parameter, but predicted in terms of the other model parameters. Higher-order corrections are crucial for a precise prediction of $M_{h}$, see refs. [120-122] for reviews.

The spectacular discovery of a Higgs boson at ATLAS and CMS, as announced in July $2012[84,85]$ can be interpreted as the discovery of the light $\mathcal{C} \mathcal{P}$-even Higgs boson of the MSSM Higgs spectrum [123] (see also refs. [124, 125] and references therein). The experimental average for the (SM) Higgs boson mass is taken to be [126]

$$
M_{H}^{\exp }=125.1 \pm 0.3 \mathrm{GeV} .
$$

Adding a $3(2) \mathrm{GeV}$ theory uncertainty [127-129] for the Higgs boson mass calculation in the MSSM we arrive at

$$
M_{h}=125.1 \pm 3.1(2.1) \mathrm{GeV}
$$

as our allowed range.

\footnotetext{
${ }^{5}$ See also references therein.
} 
For the lightest Higgs mass prediction we used the code FeynHiggs [127, 129-136] (version 2.14.0 beta). The evaluation of Higgs boson masses within FeynHiggs is based on the combination of a Feynman-diagrammatic calculation and a resummation of the (sub)leading and logarithms contributions of the (general) type $\log \left(m_{\tilde{t}} / m_{t}\right)$ in all orders of perturbation theory. This combination ensures a reliable evaluation of $M_{h}$ also for large SUSY mass scales (see section 6 below). With respect to previous versions several refinements in the combination of the fixed order log resummed calculation have been included, see ref. [129]. They resulted not only in a more precise $M_{h}$ evaluation for high SUSY mass scales, but in particular in a downward shift of $M_{h}$ at the level of $\mathcal{O}(2 \mathrm{GeV})$ for large SUSY masses.

In our previous analysis [24] the Higgs boson mass was calculated using a "mixedscale" one-loop RG approach, which captures only the leading corrections up to two-loop order. Consequently, our new implementation of the $M_{h}$ calculation is substantially more sophisticated and in particular reliable for high stop mass scales. Furthermore, in that previous analysis no B physics constraints were used, which now pose relevant constraints on the allowed parameters space and thus on the prediction of the SUSY spectrum.

\section{$6 \quad$ Numerical analysis}

In this section we analyze the particle spectrum predicted by the reduced MSSM. So far the relations among reduced parameters in terms of the fundamental ones derived in sections 3 and 4 had a part which was RGI and a another part originating from the corrections, which are scale dependent. In our analysis here we choose the unification scale to apply the corrections to the RGI relations. It should be noted that we are assuming a covering GUT, and thus unification of the three gauge couplings, as well as a unified gaugino mass $M$ at that scale. Also to be noted is that in the dimensionless sector of the theory since $Y_{\tau}$ cannnot be reduced in favor of the fundamental parameter $\alpha_{3}$, the mass of the $\tau$ lepton is an input parameter and consequently $\rho_{\tau}$, is an independent parameter too. At low energies, we fix the values of $\rho_{\tau}$ and $\tan \beta$ using the mass of the tau lepton $m_{\tau}\left(M_{Z}\right)$. For each value of $\rho_{\tau}$ there is a corresponding value of $\tan \beta$ that gives the appropriate $m_{\tau}\left(M_{Z}\right)$. Then we use the value found for $\tan \beta$ together with $G_{t, b}$, as obtained from the reduction equations and their respective corrections, to determine the top and bottom quark masses. We require that both the bottom and top masses are within $2 \sigma$ of their experimental value, which singles out large $\tan \beta$ values, $\tan \beta \sim 42-47$. Correspondingly, in the dimensionful sector of the theory the $\rho_{h_{\tau}}$ is a free parameter, since $h_{\tau}$ cannot be reduced in favor of the fundamental parameter $M$ (the unified gaugino mass scale). $\mu$ is a free parameter, as it cannot be reduced in favor of $M_{3}$ as discussed above. On the other hand $m_{3}^{2}$ could be reduced, but here it is chosen to leave it free. However, $\mu$ and $m_{3}^{2}$ are restricted from the requirement of EWSB, and only $\mu$ is taken as an independent parameter. Finally, the other parameter in the Higgs-boson sector, the $\mathcal{C} \mathcal{P}$-odd Higgs-boson mass $M_{A}$ is evaluated from $\mu$, as well as from $m_{H_{u}}^{2}$ and $m_{H_{d}}^{2}$, which are obtained from the reduction equations. In total we vary the parameters $\rho_{\tau}, \rho_{h_{\tau}}, M$ and $\mu$. 
We start our numerical analysis with the top and the bottom quark masses. As mentioned above, the variation of $\rho_{\tau}$ yields the values of $m_{t}$ (the top pole mass) and $m_{b}\left(M_{Z}\right)$, the running bottom quark mass at the $Z$ boson mass scale, where scan points which are not within $2 \sigma$ of the experimental data are neglected. This is shown in figure 1 . The experimental values are indicated by the horizontal lines and are taken to be [109],

$$
m_{t}=173.34 \pm 1.52 \mathrm{GeV}, \quad m_{b}\left(M_{Z}\right)=2.83 \pm 0.1 \mathrm{GeV},
$$

with the uncertainties at the $2 \sigma$ level. One can see that the scan yields many parameter points that are in very good agreement with the experimental data.

We continue our numerical investigation with the analysis of the lightest MSSM Higgsboson mass. The prediction for $M_{h}$ is shown in figure 2 as a function of $M$ (the common gaugino mass at the unification scale) in the range $1 \mathrm{TeV} \lesssim M \lesssim 6 \mathrm{TeV}$. The lightest Higgs mass ranges in

$$
M_{h} \sim 124-129 \mathrm{GeV},
$$

where we discard the "spreaded" points with possibly lower masses, which result from a numerical instability in the Higgs-boson mass calculation. One should keep in mind that these predictions are subject to a theory uncertainty of $3(2) \mathrm{GeV}$, see above. The red points correspond to the full parameter scan, whereas the green points are the subset that is in agreement with the $B$-physics observables as discussed above (which do not exhibit any numerical instability). The inclusion of the flavor observables shifts the lower bound for $M_{h}$ up to $\sim 126 \mathrm{GeV}$.

The horizontal lines in figure 2 show the central value of the experimental measurement (solid), the $\pm 2.1 \mathrm{GeV}$ uncertainty (dashed) and the $\pm 3.1 \mathrm{GeV}$ uncertainty (dot-dashed). The requirement to obtain a light Higgs boson mass value in the correct range yields an upper limit on $M$ of about 5 (4) TeV for $M_{h}=125.1 \pm 2.1(3.1) \mathrm{GeV}$.

Naturally the $M_{h}$ limit also sets an upper limit on the low-energy SUSY masses. The full particle spectrum of the reduced MSSM (where we restricted ourselves as before to the third generation of sfermions) compliant with the $B$-physics observables is shown in figure 3 . In the upper (lower) plot we impose $M_{h}=125.1 \pm 3.1(2.1) \mathrm{GeV}$. Including the Higgs mass constraints in general favors the somewhat higher part of the SUSY particle mass spectra. The tighter $M_{h}$ range cuts off the very high SUSY mass scales. The lighter SUSY particles are given by the electroweak spectrum, which starts around $\sim 1.3 \mathrm{TeV}$. They will mostly remain unobservable at the LHC and at future $e^{+} e^{-}$colliders such as the ILC or CLIC, with only the very lower range mass range below $\sim 1.5 \mathrm{TeV}$ might be observable at CLIC (with $\sqrt{s}=3 \mathrm{TeV}$ ). The colored mass spectrum starts at around $\sim 4 \mathrm{TeV}$, which will remain unobservable at the (HL-)LHC. However, the colored spectrum would be accessible at the FCC-hh [139]. The same applies to the heavy Higgs-boson spectrum. The four "new" Higgs bosons will likely remain outside the reach of the (HL-)LHC, ILC and CLIC, again with the very lower part of the spectrum potentially accessible at CLIC. However, the full Higgs boson spectrum would be covered at the FCC-hh [139].

In table 1 we show three example spectra of the reduced MSSM, which span the mass range of the parameter space that is in agreement with the $B$-physics observables and the 

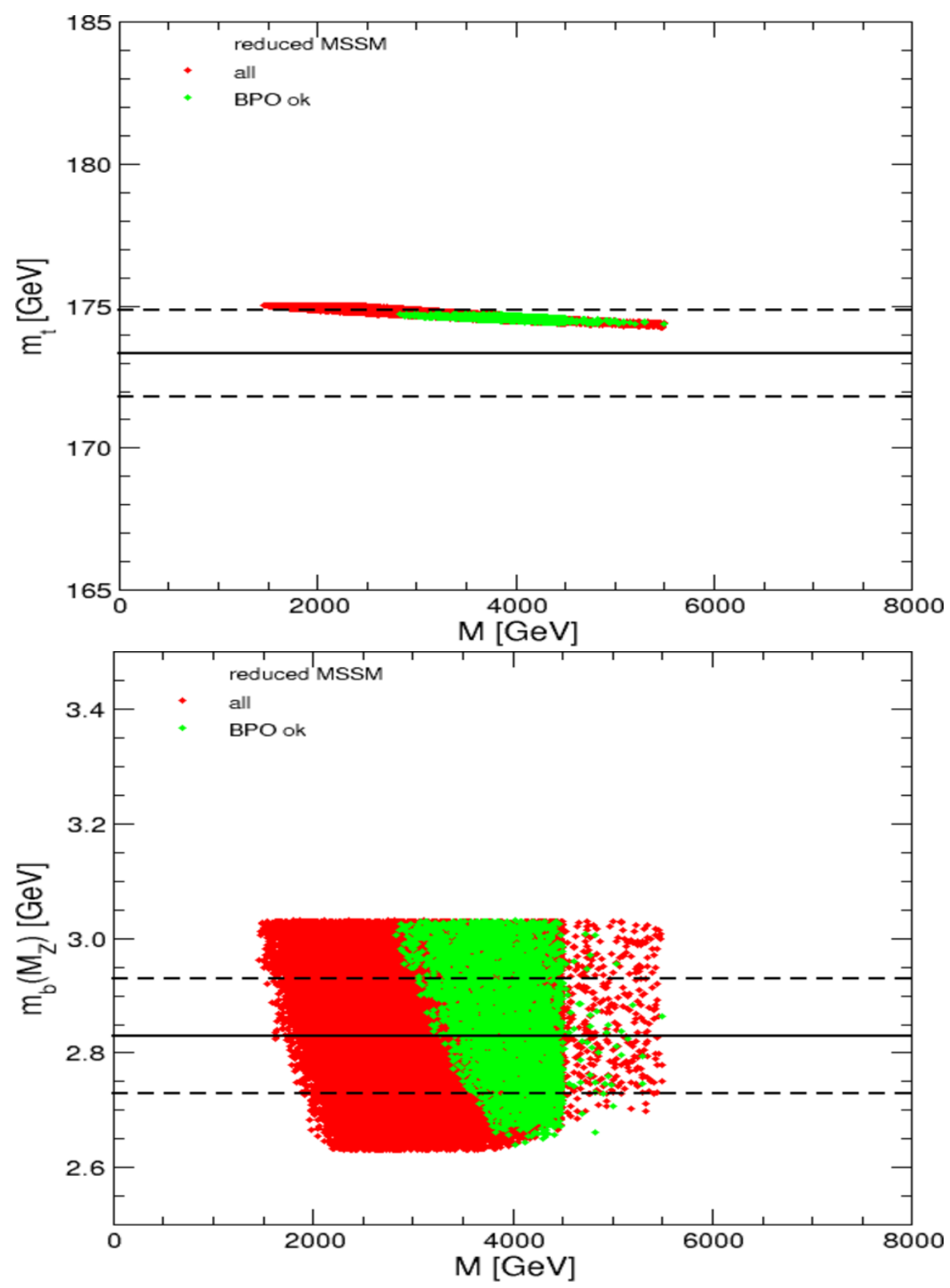

Figure 1. The upper (lower) plot shows our results within the reduced MSSM for the top (bottom) quark mass. The horizontal lines indicate the experimental values as given in eq. (6.1).

Higgs-boson mass measurement. The four Higgs boson masses are denoted as $M_{h}, M_{H}$, $M_{A}$ and $M_{H^{ \pm}} \cdot m_{\tilde{t}_{1,2}}, m_{\tilde{b}_{1,2}}, m_{\tilde{g}}, m_{\tilde{\tau}_{1,2}}$, are the scalar top, scalar bottom, gluino and scalar tau masses, respectively. $m_{\tilde{\chi}_{1,2}^{ \pm}}$and $m_{\tilde{\chi}_{1,2,3,4}^{0}}$ denote the chargino and neutralino masses. The rows labelled "light" correspond to the spectrum with the smallest $m_{\tilde{\chi}_{1}^{0}}$ value (which is independent of upper limit in $M_{h}$ ). This point is an example for the lowest $M_{h}$ values that we can reach in our scan. As discussed above, the heavy Higgs boson spectrum starts above 1.4 TeV, which is at the borderline of the reach of CLIC with $\sqrt{s}=3 \mathrm{TeV}$. The colored spectrum is found between $\sim 4 \mathrm{TeV}$ and $\sim 6 \mathrm{TeV}$, outside the range of the (HL-)LHC. The 


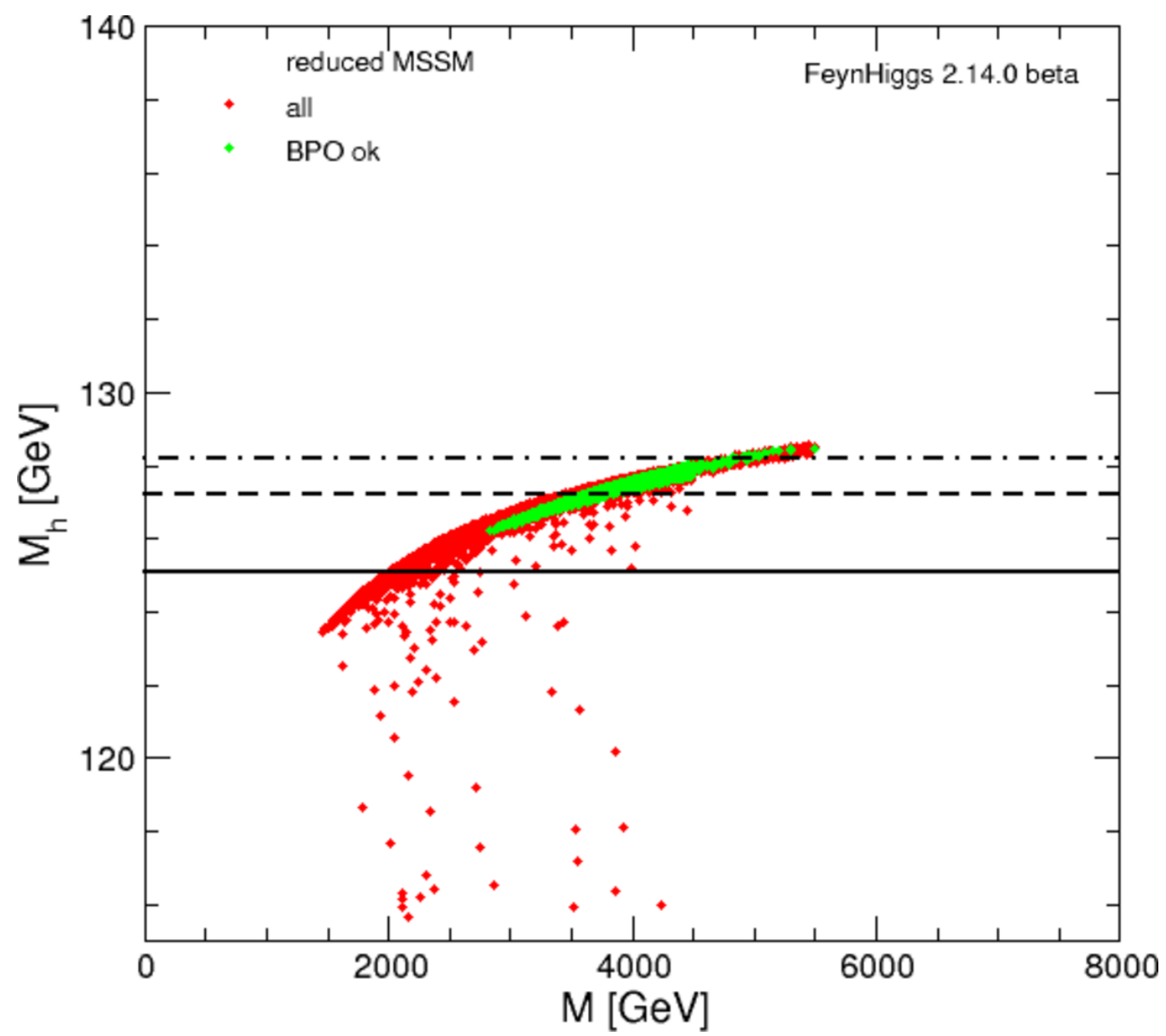

Figure 2. The lightest Higgs boson mass, $M_{h}$, as a function of $M$ (the common gaugino mass at the unification scale) in the reduced MSSM. The red points is the full model prediction. The green points fulfill the $B$-physics constraints (see text).

LSP has a mass of $m_{\tilde{\chi}_{1}^{0}}=1339$, which might offer the possibility of $e^{+} e^{-} \rightarrow \tilde{\chi}_{1}^{0} \tilde{\chi}_{1}^{0} \gamma$ at CLIC. All other electroweak particles are too heavy to be produced at CLIC or the (HL-)LHC. " $\delta M_{h}=2.1(3.1)$ " has the largest $m_{\tilde{\chi}_{1}^{0}}$ for $M_{h} \leq 125.1+2.1(3.1) \mathrm{GeV}$. While, following the mass relations in the reduced MSSM, the mass spectra are substantially heavier than in the "light" case, one can also observe that the smaller upper limit on $M_{h}$ results in substantially lower upper limits on the various SUSY and Higgs-boson masses. However, even in the case of $\delta M_{h}=2.1 \mathrm{GeV}$, all particles are outside the reach of the (HL-)LHC and CLIC. On the other hand, all spectra offer good possibilities for their discovery at the FCC-hh [139], as discussed above.

Finally, we note that with such a heavy SUSY spectrum, despite the large values of $\tan \beta$, the anomalous magnetic moment of the muon, $(g-2)_{\mu}$ (with $\left.a_{\mu} \equiv(g-2)_{\mu} / 2\right)$, gives only a negligible correction to the SM prediction. The comparison of the experimental result and the SM value shows a deviation of $\sim 3.5 \sigma$ [140-151]. Consequently, since the results would be very close to the SM results, the model has the same level of difficulty with the $a_{\mu}$ measurement as the SM. 


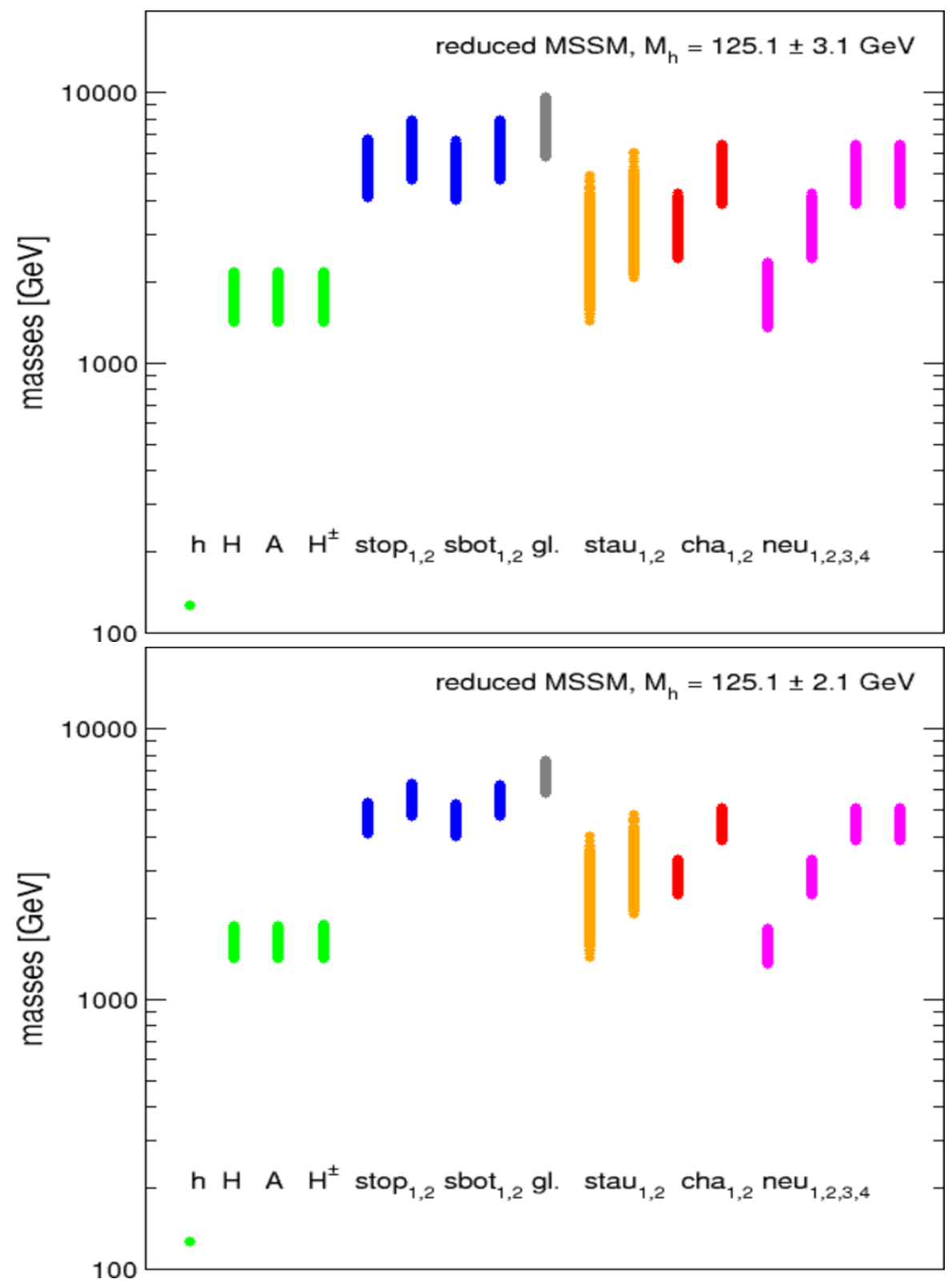

Figure 3. The upper (lower) plot shows the spectrum of the reduced MSSM after imposing the constraint $M_{h}=125.1 \pm 3.1(2.1) \mathrm{GeV}$. The points shown are in agreement with the $B$-physics observables. The light (green) points on the left are the various Higgs boson masses. The dark (blue) points following are the two scalar top and bottom masses, followed by the lighter (gray) gluino mass. Next come the lighter (beige) scalar tau masses. The darker (red) points to the right are the two chargino masses followed by the lighter shaded (pink) points indicating the neutralino masses. 


\begin{tabular}{|c|rrrrrrrrr|}
\hline & $M_{h}$ & $M_{H}$ & $M_{A}$ & $M_{H^{ \pm}}$ & $m_{\tilde{t}_{1}}$ & $m_{\tilde{t}_{2}}$ & $m_{\tilde{b}_{1}}$ & $m_{\tilde{b}_{2}}$ & $m_{\tilde{g}}$ \\
\hline light & 126.2 & 1433 & 1433 & 1446 & 4052 & 4736 & 3989 & 4723 & 5789 \\
$\delta M_{h}=2.1$ & 127.2 & 1570 & 1570 & 1572 & 5361 & 6289 & 5282 & 6279 & 7699 \\
$\delta M_{h}=3.1$ & 128.1 & 1886 & 1886 & 1888 & 6762 & 7951 & 6653 & 7943 & 9683 \\
\hline
\end{tabular}

\begin{tabular}{|c|ccccccccc|}
\hline & $m_{\tilde{\tau}_{1}}$ & $m_{\tilde{\tau}_{2}}$ & $m_{\tilde{\chi}_{1}^{ \pm}}$ & $m_{\tilde{\chi}_{2}^{ \pm}}$ & $m_{\tilde{\chi}_{1}^{0}}$ & $m_{\tilde{\chi}_{2}^{0}}$ & $m_{\tilde{\chi}_{3}^{0}}$ & $m_{\tilde{\chi}_{4}^{0}}$ & $\tan \beta$ \\
\hline light & 1906 & 2066 & 2430 & 3867 & 1339 & 2430 & 3864 & 3866 & 42.6 \\
$\delta M_{h}=2.1$ & 1937 & 2531 & 3299 & 5166 & 1833 & 3299 & 5114 & 5116 & 43.1 \\
$\delta M_{h}=3.1$ & 3153 & 3490 & 4248 & 6464 & 2376 & 4248 & 6462 & 6464 & 45.2 \\
\hline
\end{tabular}

Table 1. Three example spectra of the reduced MSSM. "light" has the smallest $\tilde{\chi}_{1}^{0}$ in our sample, " $\delta M_{h}=2.1(3.1)$ " has the largest $m_{\tilde{\chi}_{1}^{0}}$ for $M_{h} \leq 125.1+2.1(3.1) \mathrm{GeV}$. All masses are in GeV and rounded to 1 (0.1) $\mathrm{GeV}$ (for the light Higgs mass).

To summarize, the reduced MSSM naturally results in a light Higgs boson in the mass range measured at the LHC. On the other hand, the rest of the spectrum will remain (likely) unaccessible at the (HL-)LHC, ILC and CLIC, where such a heavy spectrum also results in SM-like light Higgs boson, in agreement with LHC measurements [152]. In other words, the model is naturally in full agreement with all LHC measurements. It can be tested definitely at the FCC-hh, where large parts of the spectrum would be in the kinematic reach.

\section{Conclusions}

In the present paper we have examined the reduced MSSM, in which we first calculate the exact relations among soft scalar and gaugino masses at the unification scale. This constitutes an interesting improvement w.r.t. previous analyses [24], which relied on the existence of a "sum rule" among soft scalar and gaugino masses, where due to the "simple" nature of the constraint agreement with experimental data could be realized more easily. It should be noted that in the reduced MSSM the "sum rule" still is valid. However, here we have the exact relations among these masses, and consequently the dimensionful SSB mass relations are as those among the dimensionless couplings.

In our phenomenological analysis we have derived the spectrum of the reduced MSSM as a function of the common gaugino mass at the GUT scale. The light Higgs boson mass was evaluated with the latest (preliminary) version of FeynHiggs [129], which yields more reliable results in the case of very large SUSY mass scales, as it turns out to be the case in our analysis. The resulting spectrum was confronted with various $B$-physics constraints. We find that the lightest Higgs mass is in very good agreement with the measured value and its experimental and theoretical uncertainties. The SUSY Higgs boson mass scale is found above $\sim 1.3 \mathrm{TeV}$, rendering the light MSSM Higgs boson SM-like, in perfect agreement 
with the experimental data. The electroweak SUSY spectrum starts at $1.3 \mathrm{TeV}$ and the colored spectrum at $\sim 4 \mathrm{TeV}$. Consequently, the reduced MSSM is in natural agreement with all LHC measurements and searches. The SUSY and heavy Higgs particles will likely escape the detection at the LHC, as well as at ILC and CLIC. On the other hand, the FCC-hh will be able to fully test the predicted parameter space.

\section{Acknowledgments}

We thank H. Bahl, T. Hahn, W. Hollik, D. Lüst and E. Seiler for helpful discussions. The work of S.H. is supported in part by the MEINCOP Spain under contract FPA2016-78022$\mathrm{P}$, in part by the Spanish Agencia Estatal de Investigación (AEI) and the EU Fondo Europeo de Desarrollo Regional (FEDER) through the project FPA2016-78645-P, and in part by the AEI through the grant IFT Centro de Excelencia Severo Ochoa SEV-2016-0597. The work of M.M. is supported partly by UNAM PAPIIT grant IN111518. The work of N.T. and G.Z. are supported by the COST actions CA15108 and CA16201. N.T. and G.Z. thank the CERN TH Department for their hospitality. G.Z. thanks the MPI Munich for hospitality.

Finally, we would like to acknowledge the very constructive discussion with the referee, delving us even deeper into the problem.

Open Access. This article is distributed under the terms of the Creative Commons Attribution License (CC-BY 4.0), which permits any use, distribution and reproduction in any medium, provided the original author(s) and source are credited.

\section{References}

[1] J.C. Pati and A. Salam, Is baryon number conserved?, Phys. Rev. Lett. 31 (1973) 661 [INSPIRE].

[2] H. Georgi and S.L. Glashow, Unity of all elementary particle forces, Phys. Rev. Lett. 32 (1974) 438 [INSPIRE].

[3] H. Georgi, H.R. Quinn and S. Weinberg, Hierarchy of interactions in unified gauge theories, Phys. Rev. Lett. 33 (1974) 451 [INSPIRE].

[4] H. Fritzsch and P. Minkowski, Unified interactions of leptons and hadrons, Annals Phys. 93 (1975) 193 [INSPIRE].

[5] H. Georgi, The state of the art - gauge theories, in Particles and fields, Williamsburg 1974, C.E. Carlson ed., American Institute of Physics, New York, U.S.A., (1974) [AIP Conf. Proc. 23 (1975) 575] [INSPIRE].

[6] U. Amaldi, W. de Boer and H. Furstenau, Comparison of grand unified theories with electroweak and strong coupling constants measured at LEP, Phys. Lett. B 260 (1991) 447 [INSPIRE].

[7] S. Dimopoulos and H. Georgi, Softly broken supersymmetry and SU(5), Nucl. Phys. B 193 (1981) 150 [INSPIRE].

[8] N. Sakai, Naturalness in supersymmetric GUTs, Z. Phys. C 11 (1981) 153 [INSPIRE]. 
[9] A.J. Buras, J.R. Ellis, M.K. Gaillard and D.V. Nanopoulos, Aspects of the grand unification of strong, weak and electromagnetic interactions, Nucl. Phys. B 135 (1978) 66 [InSPIRE].

[10] P. Fayet, Spontaneous generation of massive multiplets and central charges in extended supersymmetric theories, Nucl. Phys. B 149 (1979) 137 [INSPIRE].

[11] J. Kubo, M. Mondragón, M. Olechowski and G. Zoupanos, Testing gauge Yukawa unified models by $M_{t}$, Nucl. Phys. B 479 (1996) 25 [hep-ph/9512435] [INSPIRE].

[12] J. Kubo, M. Mondragón and G. Zoupanos, Unification beyond GUTs: gauge Yukawa unification, Acta Phys. Polon. B 27 (1997) 3911 [hep-ph/9703289] [InSPIRE].

[13] T. Kobayashi, J. Kubo, M. Mondragón and G. Zoupanos, Exact finite and gauge-Yukawa unified theories and their predictions, Acta Phys. Polon. B 30 (1999) 2013 [InSPIRE].

[14] D. Kapetanakis, M. Mondragón and G. Zoupanos, Finite unified models, Z. Phys. C 60 (1993) 181 [hep-ph/9210218] [INSPIRE].

[15] M. Mondragón and G. Zoupanos, Finite unified theories and the top quark mass, Nucl. Phys. Proc. Suppl. 37C (1995) 98 [inSPIRE].

[16] J. Kubo, M. Mondragón and G. Zoupanos, Reduction of couplings and heavy top quark in the minimal SUSY GUT, Nucl. Phys. B 424 (1994) 291 [INSPIRE].

[17] J. Kubo, M. Mondragón, N.D. Tracas and G. Zoupanos, Gauge Yukawa unification in asymptotically nonfree theories, Phys. Lett. B 342 (1995) 155 [hep-th/9409003] [INSPIRE].

[18] J. Kubo, M. Mondragón, S. Shoda and G. Zoupanos, Gauge Yukawa unification in SO(10) SUSY GUTs, Nucl. Phys. B 469 (1996) 3 [hep-ph/9512258] [INSPIRE].

[19] J. Kubo, M. Mondragón and G. Zoupanos, Perturbative unification of soft supersymmetry breaking terms, Phys. Lett. B 389 (1996) 523 [hep-ph/9609218] [INSPIRE].

[20] E. Ma, M. Mondragón and G. Zoupanos, Finite SU $(N)^{k}$ unification, JHEP 12 (2004) 026 [hep-ph/0407236] [INSPIRE].

[21] S. Heinemeyer, E. Ma, M. Mondragón and G. Zoupanos, Finiteness in SU(3) 3 models, Fortsch. Phys. 58 (2010) 729 [INSPIRE].

[22] S. Heinemeyer, E. Ma, M. Mondragón and G. Zoupanos, Finite unification: phenomenology, J. Phys. Conf. Ser. 259 (2010) 012097 [inSPIRE].

[23] S. Heinemeyer, E. Ma, M. Mondragón and G. Zoupanos, Finite SU $(3)^{3}$ model, AIP Conf. Proc. 1200 (2010) 568 [arXiv: 0910.0501] [INSPIRE].

[24] M. Mondragón, N.D. Tracas and G. Zoupanos, Reduction of couplings in the MSSM, Phys. Lett. B 728 (2014) 51 [arXiv:1309.0996] [INSPIRE].

[25] W. Zimmermann, Reduction in the number of coupling parameters, Commun. Math. Phys. 97 (1985) 211 [INSPIRE].

[26] R. Oehme and W. Zimmermann, Relation between effective couplings for asymptotically free models, Commun. Math. Phys. 97 (1985) 569 [inSPIRE].

[27] E. Ma, Modified quantum chromodynamics. 1. Exact global color symmetry and asymptotic freedom, Phys. Rev. D 17 (1978) 623 [InSPIRE].

[28] E. Ma, Fixing the Higgs boson mass, Phys. Rev. D 31 (1985) 1143 [INSPIRE].

[29] N.-P. Chang, Eigenvalue conditions and asymptotic freedom for Higgs scalar gauge theories, Phys. Rev. D 10 (1974) 2706 [InSPIRE]. 
[30] S. Nandi and W.-C. Ng, Can coupling constants be related?, Phys. Rev. D 20 (1979) 972 [INSPIRE].

[31] C. Lucchesi, O. Piguet and K. Sibold, Necessary and sufficient conditions for all order vanishing $\beta$-functions in supersymmetric Yang-Mills theories, Phys. Lett. B 201 (1988) 241 [INSPIRE].

[32] C. Lucchesi, O. Piguet and K. Sibold, Vanishing $\beta$-functions in $N=1$ supersymmetric gauge theories, Helv. Phys. Acta 61 (1988) 321 [InSPIRE].

[33] C. Lucchesi and G. Zoupanos, All order finiteness in $N=1 S Y M$ theories: criteria and applications, Fortsch. Phys. 45 (1997) 129 [hep-ph/9604216] [InSPIRE].

[34] A.V. Ermushev, D.I. Kazakov and O.V. Tarasov, Finite $N=1$ supersymmetric grand unified theories, Nucl. Phys. B 281 (1987) 72 [inSPIRE].

[35] D.I. Kazakov, Finite N=1 SUSY gauge field theories, Mod. Phys. Lett. A 2 (1987) 663 [INSPIRE].

[36] I. Jack and D.R.T. Jones, Renormalization group invariance and universal soft supersymmetry breaking, Phys. Lett. B 349 (1995) 294 [hep-ph/9501395] [INSPIRE].

[37] Y. Kawamura, T. Kobayashi and J. Kubo, Soft scalar mass sum rule in gauge Yukawa unified models and its superstring interpretation, Phys. Lett. B 405 (1997) 64 [hep-ph/9703320] [INSPIRE].

[38] J. Hisano and M.A. Shifman, Exact results for soft supersymmetry breaking parameters in supersymmetric gauge theories, Phys. Rev. D 56 (1997) 5475 [hep-ph/9705417] [INSPIRE].

[39] I. Jack and D.R.T. Jones, The gaugino $\beta$-function, Phys. Lett. B 415 (1997) 383 [hep-ph/9709364] [INSPIRE].

[40] L.V. Avdeev, D.I. Kazakov and I.N. Kondrashuk, Renormalizations in softly broken SUSY gauge theories, Nucl. Phys. B 510 (1998) 289 [hep-ph/9709397] [InSPIRE].

[41] D.I. Kazakov, Exploring softly broken SUSY theories via Grassmannian Taylor expansion, Phys. Lett. B 449 (1999) 201 [hep-ph/9812513] [InSPIRE].

[42] D.I. Kazakov, Finiteness of soft terms in finite $N=1$ SUSY gauge theories, Phys. Lett. B 421 (1998) 211 [hep-ph/9709465] [INSPIRE].

[43] I. Jack, D.R.T. Jones and A. Pickering, Renormalization invariance and the soft $\beta$-functions, Phys. Lett. B 426 (1998) 73 [hep-ph/9712542] [InSPIRE].

[44] T. Kobayashi, J. Kubo and G. Zoupanos, Further all loop results in softly broken supersymmetric gauge theories, Phys. Lett. B 427 (1998) 291 [hep-ph/9802267] [INSPIRE].

[45] Y. Yamada, Two loop renormalization group equations for soft SUSY breaking scalar interactions: supergraph method, Phys. Rev. D 50 (1994) 3537 [hep-ph/9401241] [INSPIRE].

[46] R. Delbourgo, Superfield perturbation theory and renormalization, Nuovo Cim. A 25 (1975) 646 [INSPIRE].

[47] A. Salam and J.A. Strathdee, Feynman rules for superfields, Nucl. Phys. B 86 (1975) 142 [INSPIRE].

[48] K. Fujikawa and W. Lang, Perturbation calculations for the scalar multiplet in a superfield formulation, Nucl. Phys. B 88 (1975) 61 [INSPIRE]. 
[49] M.T. Grisaru, W. Siegel and M. Roček, Improved methods for supergraphs, Nucl. Phys. B 159 (1979) 429 [INSPIRE].

[50] L. Girardello and M.T. Grisaru, Soft breaking of supersymmetry, Nucl. Phys. B 194 (1982) 65 [INSPIRE].

[51] D.R.T. Jones, L. Mezincescu and Y.P. Yao, Soft breaking of two loop finite $N=1$ supersymmetric gauge theories, Phys. Lett. B 148 (1984) 317 [INSPIRE].

[52] I. Jack and D.R.T. Jones, Soft supersymmetry breaking and finiteness, Phys. Lett. B 333 (1994) 372 [hep-ph/9405233] [INSPIRE].

[53] L.E. Ibáñez and D. Lüst, Duality anomaly cancellation, minimal string unification and the effective low-energy Lagrangian of $4 D$ strings, Nucl. Phys. B 382 (1992) 305 [hep-th/9202046] [INSPIRE].

[54] V.S. Kaplunovsky and J. Louis, Model independent analysis of soft terms in effective supergravity and in string theory, Phys. Lett. B 306 (1993) 269 [hep-th/9303040] [INSPIRE].

[55] A. Brignole, L.E. Ibáñez and C. Muñoz, Towards a theory of soft terms for the supersymmetric Standard Model, Nucl. Phys. B 422 (1994) 125 [Erratum ibid. B 436 (1995) 747] [hep-ph/9308271] [INSPIRE].

[56] J.A. Casas, A. Lleyda and C. Muñoz, Problems for supersymmetry breaking by the dilaton in strings from charge and color breaking, Phys. Lett. B 380 (1996) 59 [hep-ph/9601357] [INSPIRE].

[57] T. Kobayashi, J. Kubo, M. Mondragón and G. Zoupanos, Constraints on finite soft supersymmetry breaking terms, Nucl. Phys. B 511 (1998) 45 [hep-ph/9707425] [INSPIRE].

[58] V.A. Novikov, M.A. Shifman, A.I. Vainshtein and V.I. Zakharov, Instanton effects in supersymmetric theories, Nucl. Phys. B 229 (1983) 407 [INSPIRE].

[59] V.A. Novikov, M.A. Shifman, A.I. Vainshtein and V.I. Zakharov, $\beta$-function in supersymmetric gauge theories: instantons versus traditional approach, Phys. Lett. B 166 (1986) 329 [Sov. J. Nucl. Phys. 43 (1986) 294] [Yad. Fiz. 43 (1986) 459] [inSPIRE].

[60] M.A. Shifman, Little miracles of supersymmetric evolution of gauge couplings, Int. J. Mod. Phys. A 11 (1996) 5761 [hep-ph/9606281] [INSPIRE].

[61] K. Sibold ed., Reduction of couplings and its application in particle physics. Finite theories. Higgs and top mass predictions, PoS(Higgs \& top) 001.

[62] S. Heinemeyer et al., Reduction of couplings and its application in particle physics, Finite theories, Higgs and top mass predictions, arXiv:1411.7155 [INSPIRE].

[63] G. Zoupanos, Quantum reduction of couplings in finite unified theories, AIP Conf. Proc. 805 (2006) 287 [INSPIRE].

[64] M. Mondragón and G. Zoupanos, Finite unified theories, J. Phys. Conf. Ser. 171 (2009) 012095 [INSPIRE].

[65] M. Mondragón and G. Zoupanos, Unified gauge theories and reduction of couplings: from finiteness to fuzzy extra dimensions, SIGMA 4 (2008) 026 [arXiv:0802.3454] [INSPIRE].

[66] M. Mondragón and G. Zoupanos, New challenges in unified theories: from finiteness to fuzzy extra dimensions, Ann. U. Craiova Phys. 18 (2008) S1 [InSPIRE]. 
[67] M. Mondragón and G. Zoupanos, Finite unified theories and the prediction of the Higgs mass, Int. J. Mod. Phys. A 22 (2007) 6057 [inSPIRE].

[68] M. Mondragón and G. Zoupanos, Finite unified theories and low energy phenomenology, AIP Conf. Proc. 917 (2007) 268 [inSPIRE].

[69] M. Mondragón and G. Zoupanos, Unified theories: from finiteness to fuzzy extra dimensions, Prog. Theor. Phys. Suppl. 171 (2007) 144 [InSPIRE].

[70] M. Mondragón and G. Zoupanos, Higgs mass prediction in finite unified theories, Acta Phys. Polon. B 34 (2003) 5459 [inSPIRE].

[71] S. Heinemeyer, M. Mondragón and G. Zoupanos, Confronting finite unified theories with low-energy phenomenology, JHEP 07 (2008) 135 [arXiv:0712.3630] [INSPIRE].

[72] S. Heinemeyer, M. Mondragón and G. Zoupanos, The LHC Higgs boson discovery: implications for finite unified theories, Int. J. Mod. Phys. A 29 (2014) 1430032 [arXiv:1412.5766] [INSPIRE].

[73] S. Heinemeyer, M. Mondragón and G. Zoupanos, Finite theories predictions vs. the discovery of a Higgs-like boson at the LHC, EPJ Web Conf. 70 (2014) 00005 [INSPIRE].

[74] S. Heinemeyer, M. Mondragón and G. Zoupanos, Finite theories before and after the discovery of a Higgs boson at the LHC, Fortsch. Phys. 61 (2013) 969 [arXiv:1305.5073] [INSPIRE].

[75] S. Heinemeyer, M. Mondragón and G. Zoupanos, Finite unified theories and their predicitions, Phys. Part. Nucl. 44 (2013) 299 [INSPIRE].

[76] S. Heinemeyer, M. Mondragón and G. Zoupanos, Finite theories after the discovery of a Higgs-like boson at the LHC, Phys. Lett. B 718 (2013) 1430 [arXiv:1211.3765] [InSPIRE].

[77] S. Heinemeyer, M. Mondragón and G. Zoupanos, Finite unification: theory, models and predictions, in $7^{\text {th }}$ International Spring School and Workshop on Quantum Field Theory and Hamiltonian Systems, Craiova \& Calimanesti, Romania, 10-15 May 2010, Ann. U. Craiova Phys. 21 (2011) S54 [arXiv:1101.2476] [inSPIRE].

[78] S. Heinemeyer, M. Mondragón and G. Zoupanos, Finite unification: theory and predictions, SIGMA 6 (2010) 049 [arXiv: 1001.0428] [INSPIRE].

[79] S. Heinemeyer, M. Mondragón and G. Zoupanos, Phenomenology of SU(5) finite unified theories, J. Phys. Conf. Ser. 171 (2009) 012096 [inSPIRE].

[80] S. Heinemeyer, M. Mondragón and G. Zoupanos, Selecting finite unified theories with current data, AIP Conf. Proc. 1078 (2009) 247 [arXiv:0809.2397] [INSPIRE].

[81] A. Djouadi, S. Heinemeyer, M. Mondragón and G. Zoupanos, Finite unified theories and the Higgs mass prediction, Springer Proc. Phys. 98 (2005) 273 [hep-ph/0404208] [INSPIRE].

[82] S. Heinemeyer, M. Mondragón, N. Tracas and G. Zoupanos, Reduction of couplings in quantum field theories with applications in finite theories and the MSSM, Springer Proc. Math. Stat. 111 (2014) 177 [arXiv:1403.7384] [INSPIRE].

[83] S. Heinemeyer, M. Mondragón, N. Tracas and G. Zoupanos, Reduction of couplings in a finite GUT and the MSSM, Phys. Part. Nucl. Lett. 11 (2014) 910 [inSPIRE].

[84] ATLAS collaboration, Observation of a new particle in the search for the Standard Model Higgs boson with the ATLAS detector at the LHC, Phys. Lett. B 716 (2012) 1 [arXiv: 1207.7214] [INSPIRE]. 
[85] CMS collaboration, Observation of a new boson at a mass of $125 \mathrm{GeV}$ with the CMS experiment at the LHC, Phys. Lett. B 716 (2012) 30 [arXiv:1207.7235] [INSPIRE].

[86] O. Piguet and K. Sibold, Reduction of couplings in the presence of parameters, Phys. Lett. B 229 (1989) 83 [INSPIRE].

[87] P. Breitenlohner and D. Maison, Gauge and mass parameter dependence of renormalized Green's functions, Commun. Math. Phys. 219 (2001) 179 [inSPIRE].

[88] W. Zimmermann, Scheme independence of the reduction principle and asymptotic freedom in several couplings, Commun. Math. Phys. 219 (2001) 221 [INSPIRE].

[89] J. Kubo, K. Sibold and W. Zimmermann, Higgs and top mass from reduction of couplings, Nucl. Phys. B 259 (1985) 331 [INSPIRE].

[90] B. Pendleton and G.G. Ross, Mass and mixing angle predictions from infrared fixed points, Phys. Lett. B 98 (1981) 291 [INSPIRE].

[91] J. Kubo, K. Sibold and W. Zimmermann, New results in the reduction of the Standard Model, Phys. Lett. B 220 (1989) 185 [InSPIRE].

[92] M. Misiak et al., Estimate of $\mathcal{B}\left(\bar{B} \rightarrow X_{s} \gamma\right)$ at $O\left(\alpha_{s}^{2}\right)$, Phys. Rev. Lett. 98 (2007) 022002 [hep-ph/0609232] [INSPIRE].

[93] M. Ciuchini, G. Degrassi, P. Gambino and G.F. Giudice, Next-to-leading QCD corrections to $B \rightarrow X_{s} \gamma$ in supersymmetry, Nucl. Phys. B 534 (1998) 3 [hep-ph/9806308] [INSPIRE].

[94] G. Degrassi, P. Gambino and G.F. Giudice, $B \rightarrow X_{s} \gamma$ in supersymmetry: large contributions beyond the leading order, JHEP 12 (2000) 009 [hep-ph/0009337] [INSPIRE].

[95] M. Carena, D. Garcia, U. Nierste and C.E.M. Wagner, $b \rightarrow s \gamma$ and supersymmetry with large $\tan \beta$, Phys. Lett. B 499 (2001) 141 [hep-ph/0010003] [INSPIRE].

[96] G. D'Ambrosio, G.F. Giudice, G. Isidori and A. Strumia, Minimal flavor violation: an effective field theory approach, Nucl. Phys. B 645 (2002) 155 [hep-ph/0207036] [INSPIRE].

[97] Heavy Flavor Averaging Group collaboration, D. Asner et al., Averages of b-hadron, c-hadron and $\tau$-lepton properties, arXiv:1010.1589 [INSPIRE].

[98] HFLAV: B lifetime and oscillation parameters webpage, http://www.slac.stanford.edu/xorg/hfag/osc.

[99] C. Bobeth, M. Gorbahn, T. Hermann, M. Misiak, E. Stamou and M. Steinhauser, $B_{s, d} \rightarrow \ell^{+} \ell^{-}$in the Standard Model with reduced theoretical uncertainty, Phys. Rev. Lett. 112 (2014) 101801 [arXiv:1311.0903] [INSPIRE].

[100] T. Hermann, M. Misiak and M. Steinhauser, Three-loop QCD corrections to $B_{s} \rightarrow \mu^{+} \mu^{-}$, JHEP 12 (2013) 097 [arXiv: 1311.1347] [INSPIRE].

[101] C. Bobeth, M. Gorbahn and E. Stamou, Electroweak corrections to $B_{s, d} \rightarrow \ell^{+} \ell^{-}$, Phys. Rev. D 89 (2014) 034023 [arXiv:1311.1348] [INSPIRE].

[102] A.J. Buras, Relations between $\Delta M_{s, d}$ and $B_{s, d} \rightarrow \mu \bar{\mu}$ in models with minimal flavor violation, Phys. Lett. B 566 (2003) 115 [hep-ph/0303060] [INSPIRE].

[103] G. Isidori and D.M. Straub, Minimal flavour violation and beyond, Eur. Phys. J. C 72 (2012) 2103 [arXiv: 1202.0464] [INSPIRE]. 
[104] LHCb collaboration, Measurement of the $B_{s}^{0} \rightarrow \mu^{+} \mu^{-}$branching fraction and search for $B^{0} \rightarrow \mu^{+} \mu^{-}$decays at the LHCb experiment, Phys. Rev. Lett. 111 (2013) 101805 [arXiv:1307.5024] [INSPIRE].

[105] CMS collaboration, Measurement of the $B_{s}^{0} \rightarrow \mu^{+} \mu^{-}$branching fraction and search for $B^{0} \rightarrow \mu^{+} \mu^{-}$with the CMS experiment, Phys. Rev. Lett. 111 (2013) 101804 [arXiv: 1307.5025] [INSPIRE].

[106] LHCb and CMS collaborations, Combination of results on the rare decays $B_{(s)}^{0} \rightarrow \mu^{+} \mu^{-}$ from the CMS and LHCb experiments, LHCb-CONF-2013-012, CERN, Geneva, Switzerland, (2014) [CMS-PAS-BPH-13-007].

[107] G. Isidori and P. Paradisi, Hints of large $\tan \beta$ in flavour physics, Phys. Lett. B 639 (2006) 499 [hep-ph/0605012] [INSPIRE].

[108] G. Isidori, F. Mescia, P. Paradisi and D. Temes, Flavour physics at large $\tan \beta$ with a Bino-like LSP, Phys. Rev. D 75 (2007) 115019 [hep-ph/0703035] [InSPIRE].

[109] Particle Data Group collaboration, K.A. Olive et al., Review of particle physics, Chin. Phys. C 38 (2014) 090001 [INSPIRE].

[110] A.J. Buras, P. Gambino, M. Gorbahn, S. Jager and L. Silvestrini, $\epsilon^{\prime} / \epsilon$ and rare $K$ and $B$ decays in the MSSM, Nucl. Phys. B 592 (2001) 55 [hep-ph/0007313] [InSPIRE].

[111] LHCb collaboration, Precision measurement of the $B_{s}^{0}-\bar{B}_{s}^{0}$ oscillation frequency with the decay $B_{s}^{0} \rightarrow D_{s}^{-} \pi^{+}$, New J. Phys. 15 (2013) 053021 [arXiv:1304.4741] [InSPIRE].

[112] H. Goldberg, Constraint on the photino mass from cosmology, Phys. Rev. Lett. 50 (1983) 1419 [Erratum ibid. 103 (2009) 099905] [INSPIRE].

[113] J.R. Ellis, J.S. Hagelin, D.V. Nanopoulos, K.A. Olive and M. Srednicki, Supersymmetric relics from the big bang, Nucl. Phys. B 238 (1984) 453 [INSPIRE].

[114] H.K. Dreiner, An introduction to explicit R-parity violation, hep-ph/9707435 [INSPIRE].

[115] G. Bhattacharyya, A brief review of R-parity violating couplings, in Beyond the desert 1997: accelerator and non-accelerator approaches. Proceedings, $1^{\text {st }}$ International Conference on Particle Physics beyond the Standard Model, Ringberg Castle, Tegernsee, Germany, 8-14 June 1997, pg. 194 [hep-ph/9709395] [INSPIRE].

[116] B.C. Allanach, A. Dedes and H.K. Dreiner, Bounds on R-parity violating couplings at the weak scale and at the GUT scale, Phys. Rev. D 60 (1999) 075014 [hep-ph/9906209] [INSPIRE].

[117] J.C. Romao and J.W.F. Valle, Neutrino masses in supersymmetry with spontaneously broken R parity, Nucl. Phys. B 381 (1992) 87 [INSPIRE].

[118] D.H. Lyth and E.D. Stewart, Thermal inflation and the moduli problem, Phys. Rev. D 53 (1996) 1784 [hep-ph/9510204] [INSPIRE].

[119] G.B. Gelmini and P. Gondolo, Neutralino with the right cold dark matter abundance in (almost) any supersymmetric model, Phys. Rev. D 74 (2006) 023510 [hep-ph/0602230] [INSPIRE].

[120] S. Heinemeyer, MSSM Higgs physics at higher orders, Int. J. Mod. Phys. A 21 (2006) 2659 [hep-ph/0407244] [INSPIRE].

[121] A. Djouadi, The anatomy of electro-weak symmetry breaking. II. The Higgs bosons in the minimal supersymmetric model, Phys. Rept. 459 (2008) 1 [hep-ph/0503173] [INSPIRE]. 
[122] S. Heinemeyer, W. Hollik and G. Weiglein, Electroweak precision observables in the minimal supersymmetric Standard Model, Phys. Rept. 425 (2006) 265 [hep-ph/0412214] [INSPIRE].

[123] S. Heinemeyer, O. Stål and G. Weiglein, Interpreting the LHC Higgs search results in the MSSM, Phys. Lett. B 710 (2012) 201 [arXiv:1112.3026] [INSPIRE].

[124] P. Bechtle, S. Heinemeyer, O. Stål, T. Stefaniak, G. Weiglein and L. Zeune, MSSM interpretations of the LHC discovery: light or heavy Higgs?, Eur. Phys. J. C $\mathbf{7 3}$ (2013) 2354 [arXiv:1211.1955] [INSPIRE].

[125] P. Bechtle et al., The light and heavy Higgs interpretation of the MSSM, Eur. Phys. J. C 77 (2017) 67 [arXiv: 1608.00638] [inSPIRE].

[126] ATLAS and CMS collaborations, Combined measurement of the Higgs boson mass in pp collisions at $\sqrt{s}=7$ and $8 \mathrm{TeV}$ with the ATLAS and CMS experiments, Phys. Rev. Lett. 114 (2015) 191803 [arXiv: 1503.07589] [INSPIRE].

[127] G. Degrassi, S. Heinemeyer, W. Hollik, P. Slavich and G. Weiglein, Towards high precision predictions for the MSSM Higgs sector, Eur. Phys. J. C 28 (2003) 133 [hep-ph/0212020] [INSPIRE].

[128] O. Buchmueller et al., Implications of improved Higgs mass calculations for supersymmetric models, Eur. Phys. J. C 74 (2014) 2809 [arXiv:1312.5233] [INSPIRE].

[129] H. Bahl, S. Heinemeyer, W. Hollik and G. Weiglein, Reconciling EFT and hybrid calculations of the light MSSM Higgs-boson mass, Eur. Phys. J. C 78 (2018) 57 [arXiv: 1706.00346] [INSPIRE].

[130] S. Heinemeyer, W. Hollik and G. Weiglein, FeynHiggs: a program for the calculation of the masses of the neutral CP even Higgs bosons in the MSSM, Comput. Phys. Commun. 124 (2000) 76 [hep-ph/9812320] [INSPIRE].

[131] S. Heinemeyer, W. Hollik and G. Weiglein, The masses of the neutral CP-even Higgs bosons in the MSSM: accurate analysis at the two loop level, Eur. Phys. J. C 9 (1999) 343 [hep-ph/9812472] [INSPIRE].

[132] M. Frank, T. Hahn, S. Heinemeyer, W. Hollik, H. Rzehak and G. Weiglein, The Higgs boson masses and mixings of the complex MSSM in the Feynman-diagrammatic approach, JHEP 02 (2007) 047 [hep-ph/0611326] [INSPIRE].

[133] T. Hahn, S. Heinemeyer, W. Hollik, H. Rzehak and G. Weiglein, FeynHiggs: a program for the calculation of MSSM Higgs-boson observables - version 2.6.5, Comput. Phys. Commun. 180 (2009) 1426 [INSPIRE].

[134] T. Hahn, S. Heinemeyer, W. Hollik, H. Rzehak and G. Weiglein, High-precision predictions for the light CP-even Higgs boson mass of the minimal supersymmetric Standard Model, Phys. Rev. Lett. 112 (2014) 141801 [arXiv:1312.4937] [INSPIRE].

[135] H. Bahl and W. Hollik, Precise prediction for the light MSSM Higgs boson mass combining effective field theory and fixed-order calculations, Eur. Phys. J. C 76 (2016) 499 [arXiv: 1608.01880] [INSPIRE].

[136] FeynHiggs: overview webpage, http://www.feynhiggs.de.

[137] M. Carena, H.E. Haber, S. Heinemeyer, W. Hollik, C.E.M. Wagner and G. Weiglein, Reconciling the two loop diagrammatic and effective field theory computations of the mass of the lightest CP-even Higgs boson in the MSSM, Nucl. Phys. B 580 (2000) 29 [hep-ph/0001002] [INSPIRE]. 
[138] S. Heinemeyer, MSSM Higgs physics at higher orders, Int. J. Mod. Phys. A 21 (2006) 2659 [hep-ph/0407244] [INSPIRE].

[139] M. Mangano, Physics at the FCC-hh, a $100 \mathrm{TeV}$ pp collider, CERN Yellow Report CERN-2017-003-M, CERN, Geneva, Switzerland, (2017) [arXiv:1710.06353] [INSPIRE].

[140] Muon G-2 collaboration, G.W. Bennett et al., Measurement of the negative muon anomalous magnetic moment to 0.7 ppm, Phys. Rev. Lett. 92 (2004) 161802 [hep-ex/0401008] [INSPIRE].

[141] Muon G-2 collaboration, G.W. Bennett et al., Final report of the muon E821 anomalous magnetic moment measurement at BNL, Phys. Rev. D 73 (2006) 072003 [hep-ex/0602035] [INSPIRE].

[142] D. Stöckinger, The muon magnetic moment and supersymmetry, J. Phys. G 34 (2007) R45 [hep-ph/0609168] [INSPIRE].

[143] J.P. Miller, E. de Rafael and B.L. Roberts, Muon $(g-2)$ : experiment and theory, Rept. Prog. Phys. 70 (2007) 795 [hep-ph/0703049] [InSPIRE].

[144] J. Prades, E. de Rafael and A. Vainshtein, The hadronic light-by-light scattering contribution to the muon and electron anomalous magnetic moments, Adv. Ser. Direct. High Energy Phys. 20 (2009) 303 [arXiv:0901.0306] [InSPIRE].

[145] F. Jegerlehner and A. Nyffeler, The muon g-2, Phys. Rept. 477 (2009) 1 [arXiv:0902.3360] [INSPIRE].

[146] M. Davier, A. Hoecker, B. Malaescu, C.Z. Yuan and Z. Zhang, Reevaluation of the hadronic contribution to the muon magnetic anomaly using new $e^{+} e^{-} \rightarrow \pi^{+} \pi^{-}$cross section data from BABAR, Eur. Phys. J. C 66 (2010) 1 [arXiv:0908.4300] [InSPIRE].

[147] J. Prades, Standard Model prediction of the muon anomalous magnetic moment, Acta Phys. Polon. Supp. 3 (2010) 75 [arXiv:0909.2546] [InSPIRE].

[148] T. Teubner, K. Hagiwara, R. Liao, A.D. Martin and D. Nomura, Update of $g-2$ of the muon and $\Delta \alpha$, Chin. Phys. C 34 (2010) 728 [arXiv:1001.5401] [InSPIRE].

[149] M. Davier, A. Hoecker, B. Malaescu and Z. Zhang, Reevaluation of the hadronic contributions to the muon $g-2$ and to $\alpha\left(M_{Z}^{2}\right)$, Eur. Phys. J. C 71 (2011) 1515 [Erratum ibid. C 72 (2012) 1874] [arXiv: 1010.4180] [INSPIRE].

[150] F. Jegerlehner and R. Szafron, $\rho^{0}-\gamma$ mixing in the neutral channel pion form factor $F_{\pi}^{e}$ and its role in comparing $e^{+} e^{-}$with $\tau$ spectral functions, Eur. Phys. J. C 71 (2011) 1632 [arXiv:1101.2872] [INSPIRE].

[151] M. Benayoun, P. David, L. DelBuono and F. Jegerlehner, An update of the HLS estimate of the muon g-2, Eur. Phys. J. C 73 (2013) 2453 [arXiv:1210.7184] [INSPIRE].

[152] ATLAS and CMS collaborations, Measurements of the Higgs boson production and decay rates and constraints on its couplings from a combined ATLAS and CMS analysis of the LHC pp collision data at $\sqrt{s}=7$ and 8 TeV, JHEP 08 (2016) 045 [arXiv:1606.02266] [INSPIRE]. 